The experimental error in $\varphi_{0}{ }^{*}$ and $\delta^{*}$ is $\pm 0.5^{\circ}$. hydrate are:

The condition

$$
\operatorname{tg}\left[\varphi_{0 b}^{*}\right]_{a} \cdot \operatorname{tg}\left[\varphi_{0 c}^{*}\right]_{b} \cdot \operatorname{tg}\left[\varphi_{0 a}^{*}\right]_{c}=1
$$

is fulfilled within the experimental error. For the calculation of the torsional parameters by least squares fit we used only the $a$-axis rotation to suppress the strange behaviour of nonlinearity for $b$ axis rotation (compare Fig. 7). Using the value $\varphi_{n}$ and $\delta_{n}$ given for $\mathrm{Na}_{2} \mathrm{~S}_{2} \mathrm{O}_{6} \cdot 2 \mathrm{H}_{2} \mathrm{O}$ for $\mathrm{Li}_{2} \mathrm{~S}_{2} \mathrm{O}_{6} \cdot 2 \mathrm{H}_{2} \mathrm{O}$, from the $a$-axis rotation the relation

$$
\left\langle\Theta_{z}^{2}\right\rangle=0.033+0.90\left\langle\Theta_{x}^{2}\right\rangle
$$

was found. Comparing this relation for the lithium compound with the sodium result

$$
\left\langle\Theta_{z}^{2}\right\rangle=0.034+0.91\left\langle\Theta_{x}^{2}\right\rangle
$$

both expressions are identical within the experimental error of $10 \%$. Additionally the torsional frequency $\boldsymbol{v}_{x}$ of the lithium salt found by IR spectroscopy is the same as for the sodium compound. Consequently the torsional parameters, the height of the potential barrier, and the equilibrium $\mathrm{p}-\mathrm{p}$ distance for the $\mathrm{H}_{2} \mathrm{O}$ molecules of lithium dithionate di-

$$
\begin{aligned}
& \left\langle\Theta_{x}{ }^{2}\right\rangle=0.027 \pm 0.003 ; \quad\left\langle\Theta_{z}{ }^{2}\right\rangle=0.061 \pm 0.006 \\
& V_{0}=(6.0 \pm 1) \mathrm{kcal} / \mathrm{mole} \text { and } R_{\mathrm{e}}=(1.515 \pm 0.015) \AA
\end{aligned}
$$

\section{Conclusions}

The results of the ${ }^{1} \mathrm{H}-\mathrm{NMR}$ investigations on $\mathrm{Na}_{2} \mathrm{~S}_{2} \mathrm{O}_{6} \cdot 2 \mathrm{H}_{2} \mathrm{O}$ and $\mathrm{Li}_{2} \mathrm{~S}_{2} \mathrm{O}_{6} \cdot 2 \mathrm{H}_{2} \mathrm{O}$ at room temperature show that the influence of the dynamical behaviour of the water molecules in the crystals changes the value of the intramolecular $\mathrm{HH}$ distance remarkably. The influence can be studied only if other physical arguments like IR spectra or the temperature dependence of the NMR spectra are available besides the room temperature NMR experiments. The consequences of the dynamics of $\mathrm{H}_{2} \mathrm{O}$ in the crystals on the determination of the proton positions and the interpretation of the hydrogen bonds are discussed in the following paper.

Acknowledgment: We are grateful to Dr. J. M. Dereppe, Laboratoire de Chimie-Physique, Université de Louvain, Belgium, for interesting discussions on this subject.

\title{
Dynamics of Water in Crystal Hydrates
}

\author{
II. The Crystal Structure of $\mathrm{Na}_{2} \mathrm{~S}_{2} \mathrm{O}_{6} \cdot 2 \mathrm{H}_{2} \mathrm{O}$ and $\mathrm{Li}_{2} \mathrm{~S}_{2} \mathrm{O}_{6} \cdot 2 \mathrm{H}_{2} \mathrm{O}$ \\ Ingo Berthold and Alarich Weiss \\ Eduard-Zintl-Institut der Technischen Hochschule Darmstadt, Lehrstuhl für Physikalische Chemie
}

(Z. Naturforschg. 22 a, 1440—1451 [1967] ; received 24 May 1967)

\begin{abstract}
The crystal structure of $\mathrm{Na}_{2} \mathrm{~S}_{2} \mathrm{O}_{6} \cdot 2 \mathrm{H}_{2} \mathrm{O}$ has been refined by means of single crystal X-ray intensity data. The structure of the isotypic $\mathrm{Li}_{2} \mathrm{~S}_{2} \mathrm{O}_{6} \cdot 2 \mathrm{H}_{2} \mathrm{O}$ was also determined by single crystal studies. Two dimensional differential Fourier synthesis revealed the approximate positions of the hydrogen atoms. From ${ }^{1} \mathrm{H}-\mathrm{NMR}$ investigations, the crystal structure, and information gained by IR spectroscopy the complete atomic arrangement in these two substances was obtained. The influence of the dynamical behaviour of the water molecules is taken into account in determining the hydrogen positions.
\end{abstract}

Although there are already data available on the crystal structure of $\mathrm{Na}_{2} \mathrm{~S}_{2} \mathrm{O}_{6} \cdot 2 \mathrm{H}_{2} \mathrm{O}$ from $\mathrm{NMR}$ spectroscopy ${ }^{1-3}$ and X-ray analysis ${ }^{4}$, the calculation of the electric field gradient on the lattice sites of ${ }^{23} \mathrm{Na}$ and ${ }^{7} \mathrm{Li}$ nuclei demands a knowledge of the atomic coordinates as accurate as possible. Since we

1 M. van Meersche, J. M. Dereppe, and P. W. Lobo, Acta Cryst. 16, 95 [1963].

2 I. Berthold and A. Weiss, Z. Physik. Chem. Frankfurt 38, 140 [1963]. are interested in understanding the nuclear quadrupole coupling tensor of ${ }^{23} \mathrm{Na}$ in $\mathrm{Na}_{2} \mathrm{~S}_{2} \mathrm{O}_{6} \cdot 2 \mathrm{H}_{2} \mathrm{O}$ in magnitude and orientation, a redetermination of the crystal structure of $\mathrm{Na}_{2} \mathrm{~S}_{2} \mathrm{O}_{6} \cdot 2 \mathrm{H}_{2} \mathrm{O}$ was undertaken. Accordingly the interpretation of the NQR experiments on ${ }^{7} \mathrm{Li}$ in single crystals of $\mathrm{Li}_{2} \mathrm{~S}_{2} \mathrm{O}_{6} \cdot 2 \mathrm{H}_{2} \mathrm{O}$ is

3 I. Berthold and A. Weiss, Ber. Bunsenges. Physik. Chem. 68, 640 [1964].

4 S. Martinez, S. Garcia Blanco, and L. Rivoir, Acta Cryst. 9, 145 [1956]. 
only meaningful with a knowledge of the crystal structure of this substance.

Goniometric measurements ${ }^{5}$ on $\mathrm{Li}_{2} \mathrm{~S}_{2} \mathrm{O}_{6} \cdot 2 \mathrm{H}_{2} \mathrm{O}$ show, that this substance is probably isotypic with $\mathrm{Na}_{2} \mathrm{~S}_{2} \mathrm{O}_{6} \cdot 2 \mathrm{H}_{2} \mathrm{O}$. The discrepancy factor $R$ of the structure determination of $\mathrm{Na}_{2} \mathrm{~S}_{2} \mathrm{O}_{6} \cdot 2 \mathrm{H}_{2} \mathrm{O}$ by Martinez et al. ${ }^{4}$ allows small improvements in the atomic coordinates by increasing the number and accuracy of X-ray intensity data and by comparing the sodium and lithium salts. The determination of the hydrogen positions in these compounds completes the knowledge of the atomic arrangement in the lattice. Then a calculation of the electric field gradient in these crystals seems to be worthwhile.

\section{Experimental}

Large single crystals of $\mathrm{Na}_{2} \mathrm{~S}_{2} \mathrm{O}_{6} \cdot 2 \mathrm{H}_{2} \mathrm{O}$ are easy to grow from aqueous solutions. A crystal of a size of $1-2 \mathrm{~cm}$ in each dimension can be obtained within a few weeks. For X-ray analysis, small crystals elongated in the [100] direction have been chosen. The size of this prismatic crystals used for the measurement of the $\mathrm{X}$-ray intensities was $0.1 \times 0.1 \times$ $5 \mathrm{~mm}^{3}$. Since $\mathrm{Na}_{2} \mathrm{~S}_{2} \mathrm{O}_{6} \cdot 2 \mathrm{H}_{2} \mathrm{O}$ is neither hygroscopic nor unstable in air the crystals may be used without any protection. Some difficulties arise in growing good single crystals of $\mathrm{Li}_{2} \mathrm{~S}_{2} \mathrm{O}_{6} \cdot 2 \mathrm{H}_{2} \mathrm{O}$ for NMR and $\mathrm{X}$-ray investigations. $\mathrm{Li}_{2} \mathrm{~S}_{2} \mathrm{O}_{6} \cdot 2 \mathrm{H}_{2} \mathrm{O}$ is very hygroscopic. Therefore small prisms grown for the $\mathrm{X}$-ray work have been sealed into Lindemann glass capillaries under dry argon. The lithium salt has a strong tendency to include solution into the crystals. In single crystals we found an excess of $1.8-2 \%$ of water in this substance $\left(\mathrm{Li}_{2} \mathrm{~S}_{2} \mathrm{O}_{6} \cdot 2.02 \mathrm{H}_{2} \mathrm{O}\right)$ in agreement with published data ${ }^{6}$. The crystals of about $1 \mathrm{~cm}^{3}$ grown for NMR experiments are not as clear as those of $\mathrm{Na}_{2} \mathrm{~S}_{2} \mathrm{O}_{6} \cdot 2 \mathrm{H}_{2} \mathrm{O}$. The habit of the crystals depends strongly on the growth velocity. Fast grown crystals show the same habit as the sodium compound, that is, the crystals are almost isometric and show many well developed faces. Slowly grown crystals have a pseudo-octahedral habit with pronounced (111) faces.

Above $24{ }^{\circ} \mathrm{C} \mathrm{Li}_{2} \mathrm{~S}_{2} \mathrm{O}_{6} \cdot 2 \mathrm{H}_{2} \mathrm{O}$ is in equilibrium with the saturated aqueous solution of $\mathrm{Li}_{2} \mathrm{~S}_{2} \mathrm{O}_{6}$. Below $24{ }^{\circ} \mathrm{C} \quad \mathrm{Li}_{2} \mathrm{~S}_{2} \mathrm{O}_{6} \cdot 4 \mathrm{H}_{2} \mathrm{O}$ crystallizes from sa-

5 P. Groth, Chemische Kristallographie, Engelmann, Leipzig 1908, Vol. II, p. 696. turated aqueous solutions. These crystals grow in the form of pseudo-hexagonal prisms which are frequently twinned and very hygroscopic. WeIsSENBERG photographs showed that $\mathrm{Li}_{2} \mathrm{~S}_{2} \mathrm{O}_{6} \cdot 4 \mathrm{H}_{2} \mathrm{O}$ is pseudohexagonal with a fairly large unit cell. No further experiments have been done with the tetrahydrate until now. In Fig. 1 the solubility of $\mathrm{Li}_{2} \mathrm{~S}_{2} \mathrm{O}_{6}$ in water is plotted as a function of temperature, and the existence of the two phases mentioned is clearly shown.

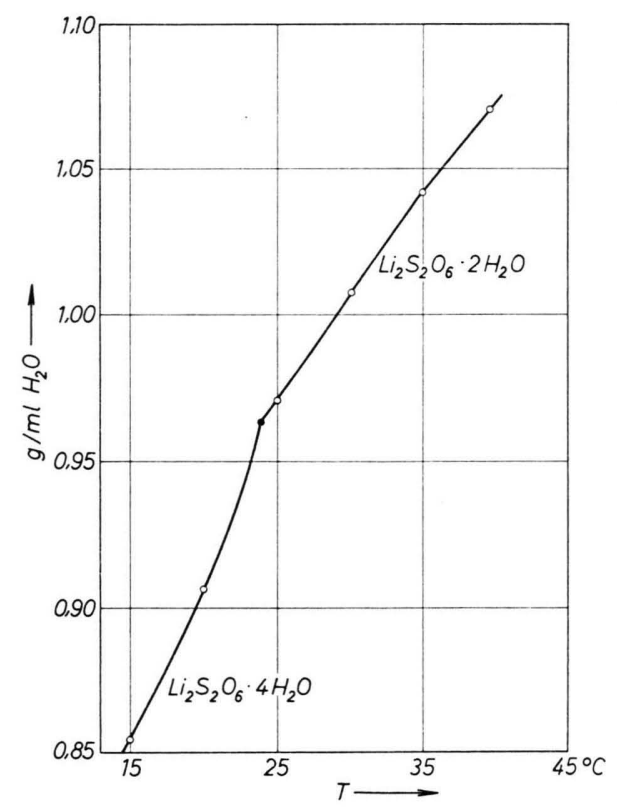

Fig. 1. The solubility of $\mathrm{Li}_{2} \mathrm{~S}_{2} \mathrm{O}_{6}$ in water as a function of the temperature.

WeIsSENBERG photographs and equi-inclination photographs were taken for the two substances. For the determination of the intensities the multiple film technique was used. The intensities have been estimated by comparing with an intensity scale in the usual way or by measurements with a photometer. The lattice constants were calculated from rotation diagrams in the asymmetric mode (Straumanis) (camera of $180 / \pi$ mm radius). Nickel filtered copper radiation $\left(\mathrm{CuK}_{\alpha}=1.5418 \AA\right.$; $\mathrm{CuK}_{a_{1}}=1.5405 \AA ; \mathrm{CuK}_{\alpha_{2}}=1.5443 \AA$ ) was used throughout the work. The parameter calculations for $\mathrm{Na}_{2} \mathrm{~S}_{2} \mathrm{O}_{6}$ $\cdot 2 \mathrm{H}_{2} \mathrm{O}$ are based on the equi-inclination photographs around [100] as rotation axis. For $\mathrm{Li}_{2} \mathrm{~S}_{2} \mathrm{O}_{6} \cdot 2 \mathrm{H}_{2} \mathrm{O}$ equiinclination photographs were taken around [100] and [010]. The relative intensities of the different reflexes cover the range from 10 to 20,000 . The crystal used for the X-ray photographs of $\mathrm{Na}_{2} \mathrm{~S}_{2} \mathrm{O}_{6} \cdot 2 \mathrm{H}_{2} \mathrm{O}$ had a

${ }^{6}$ Gmelins Handbuch der anorganischen Chemie, Verlag Chemie, Weinheim, Bergstr., 1960, 8. Aufl., Li-Erg.-Band, p. 485 . 
diameter of $0.1 \mathrm{~mm}$ with approximately cylindrical shape. X-ray photographs for $\mathrm{Li}_{2} \mathrm{~S}_{2} \mathrm{O}_{6} \cdot 2 \mathrm{H}_{2} \mathrm{O}$ around [100] as rotation axis also have been made with cylindrical crystals of about $0.1 \mathrm{~mm}$ diameter. Therefore no absorption correction was applied. The crystals of $\mathrm{Li}_{2} \mathrm{~S}_{2} \mathrm{O}_{6} \cdot 2 \mathrm{H}_{2} \mathrm{O}$ used for the photographs around [010] as rotation axis were small octahedrons of $\sim 0.6 \mathrm{~mm}$ mean diameter. In this case an absorption correction for spherical crystals with $\mu R=2.1$ was applied ${ }^{7}$. The error in the parameters calculated from the data of [010] WeissenBerg photographs is somewhat higher than from the [100] photographs, presumably through the influence of the absorption.

The scattering factors for the calculations are taken from the International Tables for X-ray Crystallography. Under the assumption of $\mathrm{Na}^{+}, \mathrm{Li}^{+}, \mathrm{O}^{-}$and $\mathrm{S}^{2+}$, $f\left(\mathrm{~S}^{2+}\right)$ was interpolated with the relation:

$$
f\left(\mathrm{~S}^{2^{+}}\right) \approx f(\mathrm{~S})-\left[f\left(\mathrm{O}^{2^{-}}\right)-f(0)\right] .
$$

The calculations necessary have been performed on a computer IBM-7090 or IBM-7094.

In accordance with MARTinez et al. ${ }^{4}$ the space group of $\mathrm{Na}_{2} \mathrm{~S}_{2} \mathrm{O}_{6} \cdot 2 \mathrm{H}_{2} \mathrm{O}$ was found to be $\mathrm{D}_{2 \mathrm{~h}}^{16}$ - Pnma. The systematic extinctions and the intensity calculations showed that $\mathrm{D}_{2 \mathrm{~h}}^{16}-\mathrm{Pnma}$ is also the space group of $\mathrm{Li}_{2} \mathrm{~S}_{2} \mathrm{O}_{6} \cdot 2 \mathrm{H}_{2} \mathrm{O}$. Table 1 contains, together with data from literature, the crystallographic data determined.

\begin{tabular}{|c|c|c|}
\hline & $\mathrm{Na}_{2} \mathrm{~S}_{2} \mathrm{O}_{6} \cdot 2 \mathrm{H}_{2} \mathrm{O}$ & $\mathrm{Li}_{2} \mathrm{~S}_{2} \mathrm{O}_{6} \cdot 2 \mathrm{H}_{2} \mathrm{O}$ \\
\hline $\begin{array}{c}\text { Formula } \\
\text { weight } \\
Z\end{array}$ & $\begin{array}{l}242.16 \\
4\end{array}$ & 210.04 \\
\hline \multicolumn{3}{|c|}{ Space } \\
\hline$\underset{a}{\text { group }}$ & $\begin{array}{l}\mathrm{D}_{2 \mathrm{~h}}^{16} \text {-Pnma } \\
6.403 \pm 0.010 \AA \AA \\
6.42 \pm 0.01 \AA\left(\text { ref. }^{4}\right)\end{array}$ & $\begin{array}{l}\mathrm{D}_{2 \mathrm{~h}}^{16} \text {-Pnma } \\
6.010 \pm 0.010 \AA\end{array}$ \\
\hline$b$ & $\begin{array}{l}10.750 \pm 0.005 \AA \\
10.75 \pm 0.01 \AA\left(\text { ref. }^{4}\right)\end{array}$ & $10.440 \pm 0.005 \AA$ \\
\hline$c$ & $10.686 \pm 0.005 \AA$ & $10.151 \pm 0.005 \AA$ \\
\hline$U$ & $735.5 \AA^{\frac{7}{3}} 0.01 \mathrm{~A}\left(\mathrm{Ite} . \mathrm{C}^{-1}\right.$ & $636.9 \AA^{3}$ \\
\hline$\varrho_{\mathbf{x}}$ & $2.186 \mathrm{~g} \cdot \mathrm{cm}^{-3}$ & $2.190 \mathrm{~g} \cdot \mathrm{cm}^{-3}$ \\
\hline Qpykn. & $2.189 \mathrm{~g} \cdot \mathrm{cm}^{-3}\left(\right.$ ref. $\left.^{5}\right)$ & $2.158 \mathrm{~g} \cdot \mathrm{cm}^{-3}\left(\right.$ ref. $\left.^{5}\right)$ \\
\hline$(a: b: c)_{\mathrm{x}}$ & $0.5956: 1: 0.9940$ & $\begin{array}{l}0.5757: 1: 0.9723 \\
0.5779: 1: 0.9657 \text { (ref. } 5 \text { ) }\end{array}$ \\
\hline & $0.5981: 1: 0.9922\left(\right.$ ref. $\left.^{5}\right)$ & $0.5779: 1: 0.9657$ (ref. 5 ) \\
\hline
\end{tabular}

Table 1. Crystallographic data.

\section{Structure Determinations}

\section{a) The Results of the X-Ray Analysis}

From the X-ray analysis of Martinez et al. ${ }^{4}$ the atomic coordinates for the heavy atoms in $\mathrm{Na}_{2} \mathrm{~S}_{2} \mathrm{O}_{6}$. $2 \mathrm{H}_{2} \mathrm{O}$ are known. We have used these coordinates

7 International Tables for X-ray Crystallography, Vol. III, The Kynoch Press, Birmingham 1962. as a basic set and with this set a least squares refinement was performed. In Table 2 the observed and calculated structure factors are given for $\mathrm{Na}_{2} \mathrm{~S}_{2} \mathrm{O}_{6}$. $2 \mathrm{H}_{2} \mathrm{O}$ and $\mathrm{Li}_{2} \mathrm{~S}_{2} \mathrm{O}_{6} \cdot 2 \mathrm{H}_{2} \mathrm{O}$, respectively.

The discrepancy factor: $R=\Sigma\left(\left|F_{0}\right|-\left|F_{\mathrm{c}}\right|\right) / \Sigma\left|F_{0}\right|$ is 0.103 for $\mathrm{Na}_{2} \mathrm{~S}_{2} \mathrm{O}_{6} \cdot 2 \mathrm{H}_{2} \mathrm{O}$, including all reflexions. The total number of reflexions observed was 477. For the individual equi-inclination photographs the following $R$ factors have been calculated: $0 k l: R=0.110$; $1 k l: R=0.090 ; 2 k l: R=0.112 ; 3 k l: R=0.100$. For $\mathrm{Li}_{2} \mathrm{~S}_{2} \mathrm{O}_{6} \cdot 2 \mathrm{H}_{2} \mathrm{O}$ the discrepancy factor is about the same as for the sodium salt. We calculated: $R=0.102$, including all reflexions. The total number of reflexions observed was 432 . The individual $R$ factors are: $0 k l$ : $R=0.104 ; \quad 1 k l: R=0.091 ; 2 k l: R=0.102 ; 3 k l$ : $R=0.117$.

Fig. 2 shows the projection of the unit cell of $\mathrm{Na}_{2} \mathrm{~S}_{2} \mathrm{O}_{6} \cdot 2 \mathrm{H}_{2} \mathrm{O}$ onto the $b c$ and $a c$ plane. The equivalent projections of the $\mathrm{Li}_{2} \mathrm{~S}_{2} \mathrm{O}_{6} \cdot 2 \mathrm{H}_{2} \mathrm{O}$ elementary cell are only slightly different by small changes in atomic coordinates and lattice constants. In Table 3 the atomic coordinates and temperature
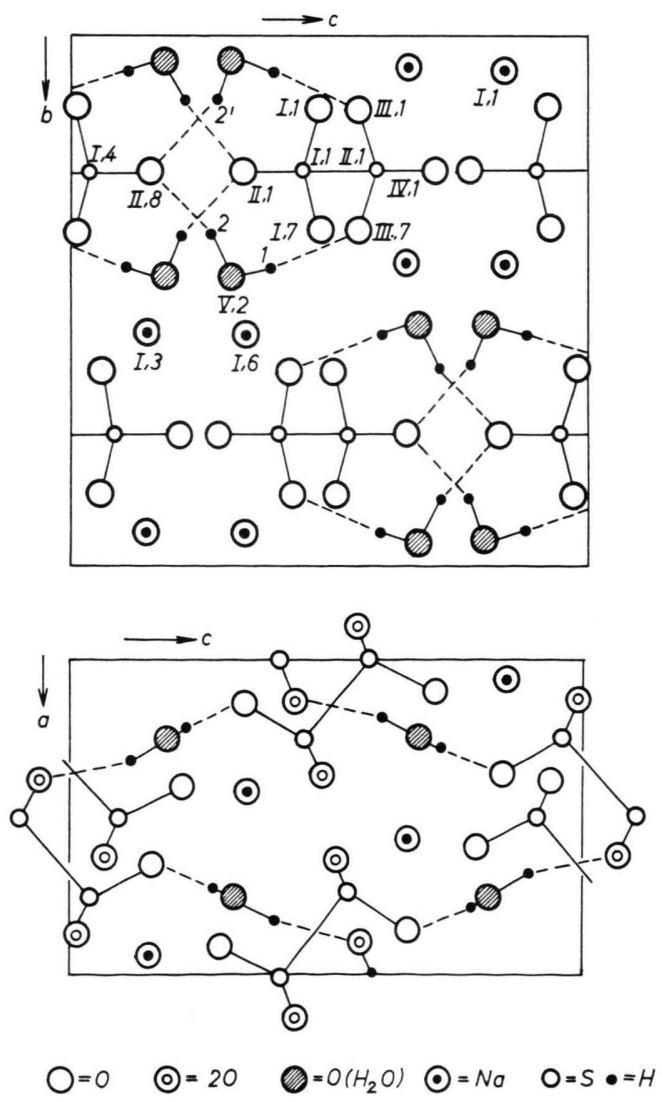

Fig. 2. Projection of the elementary cell of $\mathrm{Na}_{2} \mathrm{~S}_{2} \mathrm{O}_{6} \cdot 2 \mathrm{H}_{2} \mathrm{O}$ onto the $b c$ and $a c$ plane. 


\begin{tabular}{|c|c|c|c|c|c|c|c|c|c|c|}
\hline$h$ & $k$ & $l$ & $\left|F_{0}\right|$ & $\boldsymbol{F}_{c}$ & $\left|F_{0}\right|$ & $F_{c}$ & $h$ & $k$ & $l$ & $\left|F_{\theta}\right|$ \\
\hline 0 & 0 & 2 & 50.6 & 57.5 & 62.0 & 70.4 & 1 & 1 & 4 & 70.5 \\
\hline 0 & 0 & 4 & 17.1 & -17.3 & 53.2 & 57.4 & 1 & 1 & $\mathbf{5}$ & 42.0 \\
\hline 0 & 0 & 6 & 50.6 & 48.8 & 7.7 & 8.2 & 1 & 1 & 6 & 34.3 \\
\hline 0 & 0 & 8 & 43.5 & -43.6 & 22.9 & -21.3 & 1 & 1 & 7 & 12.7 \\
\hline 0 & 0 & 10 & 18.3 & -17.5 & 13.7 & 11.2 & 1 & 1 & 8 & 42.6 \\
\hline 0 & 1 & 1 & 38.1 & 41.3 & 32.9 & 33.2 & 1 & 1 & 9 & 5.9 \\
\hline 0 & 1 & 3 & 34.0 & 27.2 & 27.5 & 26.8 & 1 & 1 & 10 & 4.2 \\
\hline 0 & 1 & 5 & 8.4 & $\begin{array}{r}-\quad 6.9 \\
\end{array}$ & 23.6 & 17.8 & 1 & 1 & 11 & 18.1 \\
\hline 0 & 1 & 7 & 68.4 & -69.7 & 66.8 & -64.3 & 1 & 1 & 12 & 15.6 \\
\hline 0 & 1 & 9 & 48.8 & -54.9 & 46.0 & -45.2 & 1 & 1 & 13 & 14.0 \\
\hline 0 & 1 & 11 & 20.5 & -19.5 & 17.4 & -16.7 & 1 & 2 & 1 & 70.0 \\
\hline 0 & 2 & 0 & 69.7 & -74.1 & & & 1 & 2 & 2 & 53.5 \\
\hline 0 & 2 & 2 & 78.0 & -86.5 & 68.8 & -76.1 & 1 & 2 & 3 & 71.1 \\
\hline 0 & 2 & 4 & 38.3 & -32.0 & 0.0 & 1.5 & 1 & 2 & 4 & 18.4 \\
\hline 0 & $\overline{2}$ & 6 & 58.3 & 66.4 & 27.5 & 27.2 & 1 & 2 & 5 & 22.4 \\
\hline 0 & 2 & 8 & 16.6 & 10.2 & 9.5 & -7.8 & 1 & 2 & 6 & 48.9 \\
\hline 0 & 2 & 10 & 8.8 & $\begin{array}{l}-\quad 7.5 \\
-\end{array}$ & 4.3 & 4.4 & 1 & 2 & 7 & 6.4 \\
\hline 0 & 3 & 1 & 4.4 & 9.6 & 26.2 & -18.1 & 1 & 2 & 8 & 28.2 \\
\hline 0 & 3 & 3 & 49.6 & 49.0 & 38.7 & 38.1 & 1 & 2 & 9 & 5.1 \\
\hline 0 & 3 & 5 & 30.4 & -28.2 & 0.0 & 2.0 & 1 & 2 & 10 & 0.0 \\
\hline 0 & 3 & 7 & 57.8 & 55.4 & 66.5 & 69.3 & 1 & 2 & 11 & 11.8 \\
\hline 0 & 3 & 9 & 67.4 & 65.2 & 46.1 & 43.1 & 1 & 2 & 12 & 12.4 \\
\hline 0 & 3 & 11 & 38.3 & -34.4 & 11.6 & -10.1 & 1 & 2 & 13 & 19.1 \\
\hline 0 & 4 & 0 & 65.2 & 62.3 & 68.4 & 72.1 & 1 & 3 & 1 & 74.1 \\
\hline 0 & 4 & 2 & 51.2 & -56.8 & 60.2 & -73.1 & 1 & 3 & 2 & 64.8 \\
\hline 0 & 4 & $\overline{4}$ & 38.0 & -34.2 & 25.1 & -19.8 & 1 & 3 & 3 & 62.0 \\
\hline 0 & 4 & 6 & 15.1 & -11.8 & 24.6 & $-\quad 20.9$ & 1 & 3 & 4 & 28.0 \\
\hline 0 & 4 & 8 & 25.2 & -24.4 & 13.8 & -12.2 & 1 & 3 & 5 & 31.2 \\
\hline 0 & 4 & 10 & 0.0 & 1.2 & 12.9 & 12.3 & 1 & 3 & 6 & 27.5 \\
\hline 0 & 5 & 1 & 13.8 & 12.4 & 13.4 & -10.3 & 1 & 3 & 7 & 16.5 \\
\hline 0 & 5 & 3 & 2.0 & 1.9 & 10.4 & -6.3 & 1 & 3 & 8 & 17.2 \\
\hline 0 & 5 & 5 & 44.7 & $\begin{array}{l}-41.9 \\
-\end{array}$ & 27.0 & $\begin{array}{r}0.0 \\
-\quad 23.3\end{array}$ & 1 & 3 & $\begin{array}{l}9 \\
9\end{array}$ & 36.2 \\
\hline 0 & 5 & 7 & 52.0 & -50.8 & 62.5 & $\begin{array}{r}20.0 \\
-62.8\end{array}$ & 1 & 3 & 10 & 9.2 \\
\hline 0 & 5 & 9 & 0.0 & $-\quad 2.0$ & 23.3 & -23.4 & 1 & 3 & 11 & 18.6 \\
\hline 0 & 5 & 11 & 0.0 & 1.4 & 3.2 & $\begin{array}{r}20.7 \\
-\quad 3.8\end{array}$ & 1 & 3 & 12 & 0.0 \\
\hline 0 & 6 & 0 & 104.6 & -116.0 & 71.1 & $\begin{array}{r}0.0 \\
-\quad 73.8\end{array}$ & 1 & 3 & 13 & $\begin{array}{r}0.0 \\
13.6\end{array}$ \\
\hline 0 & 6 & 2 & 12.8 & 7.6 & 19.1 & $\begin{array}{r}-19.9 \\
-\end{array}$ & 1 & 4 & 1 & 49.7 \\
\hline 0 & 6 & 4 & 39.2 & 35.5 & 21.1 & 15.9 & 1 & 4 & 2 & 5.2 \\
\hline 0 & 6 & 6 & 6.0 & $\begin{array}{r}0.0 \\
-\quad 3.1\end{array}$ & 16.6 & 13.2 & 1 & 4 & 3 & 16.5 \\
\hline 0 & 6 & 8 & 20.7 & 16.5 & 0.0 & 0.7 & 1 & 4 & 4 & 13.9 \\
\hline 0 & 6 & 10 & 11.0 & 14.2 & 7.9 & 4.8 & 1 & 4 & 5 & 8.2 \\
\hline 0 & 7 & 1 & 21.0 & -19.2 & 37.8 & $\begin{array}{r}4.0 \\
-\quad 34.7\end{array}$ & 1 & 4 & 6 & 5.2 \\
\hline 0 & 7 & 3 & 12.4 & 8.1 & 0.0 & 1.9 & 1 & 4 & 7 & 0.0 \\
\hline 0 & 7 & 5 & 2.7 & 2.8 & 9.6 & 4.2 & 1 & 4 & 8 & 31.3 \\
\hline 0 & 7 & 7 & 43.3 & 41.8 & 45.8 & 42.6 & 1 & 4 & $\begin{array}{l}0 \\
9\end{array}$ & 23.0 \\
\hline 0 & 7 & 9 & 54.3 & 58.4 & 41.0 & 42.7 & 1 & 4 & 10 & 5.7 \\
\hline 0 & 7 & 11 & 8.0 & $\begin{array}{r}-\quad 5.7 \\
-\quad 1\end{array}$ & & & 1 & 4 & 11 & 22.2 \\
\hline 0 & 8 & 0 & 59.6 & 65.7 & 100.7 & 109.5 & 1 & 4 & 12 & 5.1 \\
\hline 0 & 8 & 2 & 59.1 & 72.0 & 45.9 & 49.0 & 1 & 4 & 13 & 8.8 \\
\hline 0 & 8 & 4 & 37.7 & 31.8 & 23.9 & 21.0 & 1 & $\mathbf{5}$ & 1 & $\begin{array}{r}0.0 \\
19.1\end{array}$ \\
\hline 0 & 8 & 6 & 45.9 & -43.5 & 13.8 & -13.5 & 1 & 5 & 2 & 40.2 \\
\hline 0 & 8 & 8 & 18.3 & -14.6 & 8.5 & $\begin{array}{r}-6.6 \\
-\quad 6\end{array}$ & 1 & 5 & 3 & 43.0 \\
\hline 0 & 9 & 1 & 6.1 & $\begin{array}{r}-\quad 6.1 \\
-\end{array}$ & 6.2 & $\begin{array}{r}0.0 \\
-\quad 4.6\end{array}$ & 1 & 5 & 4 & 24.6 \\
\hline 0 & 9 & 3 & 12.4 & 10.3 & 24.1 & 20.6 & 1 & 5 & 5 & 27.1 \\
\hline 0 & 9 & 5 & 8.8 & 9.7 & 0.0 & 6.1 & 1 & 5 & 6 & 0.0 \\
\hline 0 & 9 & 7 & 48.6 & -54.8 & 43.3 & -46.1 & 1 & 5 & 7 & 30.7 \\
\hline 0 & 9 & 9 & 48.7 & -44.1 & 21.6 & -26.6 & 1 & 5 & 8 & 57.0 \\
\hline 0 & 10 & 0 & 96.8 & -112.5 & 67.8 & -70.1 & 1 & 5 & 9 & 19.1 \\
\hline 0 & 10 & 2 & 24.9 & -20.3 & 30.7 & -28.5 & 1 & 5 & 10 & 0.0 \\
\hline 0 & 10 & 4 & 17.2 & 9.5 & 4.2 & -4.1 & 1 & 5 & 11 & 28.8 \\
\hline 0 & 10 & 6 & 5.5 & $\begin{array}{l}-6.2 \\
-\quad 6\end{array}$ & 8.1 & 8.3 & 1 & 5 & 12 & 21.2 \\
\hline 0 & 10 & 8 & 22.2 & 20.8 & & & 1 & 6 & 1 & 25.3 \\
\hline 0 & 11 & 1 & 20.6 & -22.1 & 18.1 & -19.4 & 1 & 6 & 2 & 29.8 \\
\hline 0 & 11 & 3 & 2.6 & $\begin{array}{r}-4.6 \\
-\quad 4\end{array}$ & 8.6 & 4.9 & 1 & 6 & 3 & 14.5 \\
\hline 0 & 11 & 5 & 23.2 & 22.9 & 7.5 & 9.8 & 1 & 6 & 4 & 12.1 \\
\hline 0 & 11 & 7 & 32.8 & 28.4 & 24.6 & 29.6 & 1 & 6 & 5 & 15.9 \\
\hline 0 & 12 & 0 & 26.4 & 27.2 & 26.8 & 30.3 & 1 & 6 & 6 & 9.0 \\
\hline 0 & 12 & 2 & 17.7 & 14.8 & & & 1 & 6 & 7 & 29.4 \\
\hline 0 & 12 & 4 & 6.5 & $\begin{array}{l}-8.0 \\
-\quad 8.0\end{array}$ & & & 1 & 6 & 8 & 30.8 \\
\hline 0 & 12 & $\begin{array}{l}\mathbf{x} \\
6\end{array}$ & $\begin{array}{r}0.0 \\
19.6\end{array}$ & -18.3 & & & 1 & 6 & 9 & 23.2 \\
\hline 0 & 13 & 1 & 4.1 & $\begin{array}{r}-5.0 \\
-\quad 5\end{array}$ & & & 1 & 6 & 10 & 2.6 \\
\hline 0 & 13 & 3 & 8.8 & $\begin{array}{r}-7.2 \\
-\quad 7.0\end{array}$ & & & 1 & 6 & 11 & 23.1 \\
\hline 1 & 0 & 2 & 57.3 & 61.1 & 51.8 & 51.6 & 1 & 6 & 12 & 3.3 \\
\hline 1 & 0 & 3 & 5.1 & $\begin{array}{r}-2.3 \\
-\quad 1\end{array}$ & 37.4 & 38.2 & 1 & 7 & 1 & 50.7 \\
\hline 1 & 0 & 4 & 91.5 & 97.2 & 85.9 & 93.8 & 1 & 7 & 2 & 49.8 \\
\hline $\begin{array}{l}1 \\
1\end{array}$ & 0 & $\begin{array}{l} \pm \\
5\end{array}$ & 56.5 & 55.9 & 53.2 & 55.1 & 1 & 7 & 3 & 12.8 \\
\hline 1 & 0 & 6 & 22.2 & 17.9 & 18.5 & 20.0 & 1 & 7 & 4 & 19.2 \\
\hline 1 & 0 & 7 & 80.0 & 88.0 & 47.7 & 52.6 & 1 & 7 & 5 & 38.6 \\
\hline 1 & 0 & 8 & 55.4 & 61.0 & 51.8 & 52.1 & 1 & 7 & 6 & 0.0 \\
\hline 1 & 0 & 9 & 15.0 & -13.5 & 0.0 & $\begin{array}{r}-2.4 \\
\end{array}$ & 1 & 7 & 7 & 0.0 \\
\hline 1 & 0 & 10 & 16.8 & 15.7 & 0.0 & 5.4 & 1 & 7 & 8 & 21.3 \\
\hline 1 & 0 & 11 & 20.6 & -22.2 & 8.4 & -6.4 & 1 & 7 & 9 & 18.6 \\
\hline 1 & 0 & 12 & 13.3 & -14.0 & 6.7 & 3.9 & 1 & 7 & 10 & 5.7 \\
\hline 1 & 0 & 13 & 0.0 & 0.2 & & & 1 & 7 & 11 & 13.2 \\
\hline 1 & 1 & 1 & 51.5 & 57.6 & 49.5 & 50.9 & 1 & 8 & 1 & 49.9 \\
\hline $\begin{array}{l}1 \\
1\end{array}$ & 1 & 2 & 43.0 & $\begin{array}{l}-41.9 \\
-\end{array}$ & 56.9 & -51.1 & 1 & 8 & 2 & 27.5 \\
\hline 1 & 1 & 3 & 52.1 & 44.3 & 36.9 & 32.4 & 1 & & 3 & 53.0 \\
\hline
\end{tabular}

Table 2. Observed and calculated structure factors $F_{h k l}$ for $\mathrm{Na}_{2} \mathrm{~S}_{2} \mathrm{O}_{6} \cdot 2 \mathrm{H}_{2} \mathrm{O}$ (A) and $\mathrm{Li}_{2} \mathrm{~S}_{2} \mathrm{O}_{6} \cdot 2 \mathrm{H}_{2} \mathrm{O}$ (B). 


\begin{tabular}{|c|c|c|c|c|c|c|}
\hline$h$ & $k$ & $l$ & $\left|F_{0}\right|$ & $F_{c}$ & $\left|F_{0}\right|$ & $F_{c}$ \\
\hline 1 & 8 & 4 & 32.8 & 33.0 & 35.5 & 37.3 \\
\hline 1 & 8 & 5 & 28.2 & 26.6 & 27.0 & 26.8 \\
\hline 1 & 8 & 6 & 30.8 & 39.3 & 23.0 & 23.2 \\
\hline 1 & 8 & 7 & 10.8 & 10.8 & 22.0 & 23.2 \\
\hline 1 & 8 & 8 & 21.1 & 22.4 & 23.0 & 24.1 \\
\hline 1 & 8 & 9 & 10.9 & 11.7 & 0.0 & 1.2 \\
\hline 1 & 8 & 10 & 2.8 & 4.1 & & \\
\hline 1 & 9 & 1 & 31.4 & 31.5 & 19.0 & 20.5 \\
\hline 1 & 9 & 2 & 33.1 & -39.8 & 26.9 & -31.7 \\
\hline 1 & 9 & 3 & 24.1 & 21.1 & 23.4 & 25.2 \\
\hline 1 & 9 & 4 & 25.8 & -26.3 & 18.9 & -21.9 \\
\hline 1 & 9 & 5 & 18.4 & 17.7 & 16.1 & 15.7 \\
\hline 1 & 9 & 6 & 5.7 & $\begin{array}{r}-5.3 \\
\end{array}$ & 0.0 & -3.5 \\
\hline 1 & 9 & 7 & 12.6 & 13.0 & 22.8 & 23.4 \\
\hline 1 & 9 & 8 & 17.2 & 17.4 & 19.7 & 18.3 \\
\hline 1 & 9 & 9 & 6.5 & $-\quad 5.9$ & 2.4 & -4.2 \\
\hline 1 & 9 & 10 & 5.0 & 5.5 & 2.4 & 7.2 \\
\hline 1 & 10 & 1 & 12.6 & 14.2 & 18.9 & 18.3 \\
\hline 1 & 10 & 2 & 17.7 & -18.8 & 22.9 & -19.8 \\
\hline 1 & 10 & 3 & 0.0 & & 13.0 & -11.5 \\
\hline 1 & 10 & 4 & 32.2 & -31.3 & 17.8 & -18.2 \\
\hline 1 & 10 & 5 & 20.3 & -22.4 & 16.9 & -19.4 \\
\hline 1 & 10 & 6 & 11.6 & -12.7 & 18.9 & -17.8 \\
\hline 1 & 10 & 7 & 28.3 & -30.3 & 16.4 & -16.4 \\
\hline 1 & 10 & 8 & 29.5 & -28.4 & 16.4 & -17.9 \\
\hline 1 & 10 & 9 & 6.2 & 7.6 & & \\
\hline 1 & 11 & 1 & 17.3 & -20.4 & 17.3 & -21.7 \\
\hline 1 & 11 & 2 & 0.0 & 1.5 & 10.1 & 12.0 \\
\hline 1 & 11 & 3 & 10.1 & -14.8 & 3.7 & -5.0 \\
\hline 1 & 11 & 5 & 8.4 & $\begin{array}{r}-\quad 9.5 \\
-\end{array}$ & 17.3 & -19.1 \\
\hline 1 & 11 & 6 & 4.4 & 4.5 & 6.1 & -4.3 \\
\hline 1 & 12 & 1 & 17.3 & -19.6 & 14.5 & -15.4 \\
\hline 1 & 12 & 2 & 14.3 & 15.3 & 14.0 & 10.5 \\
\hline 1 & 13 & 1 & 23.8 & 22.4 & 9.7 & 13.3 \\
\hline 2 & 0 & 1 & 28.1 & 32.6 & 25.0 & -22.0 \\
\hline 2 & 0 & 2 & 8.3 & $-\quad 7.3$ & 10.0 & -7.3 \\
\hline 2 & 0 & 3 & 31.8 & -35.1 & 43.3 & -49.3 \\
\hline 2 & 0 & 4 & 62.0 & -68.8 & 49.7 & -50.6 \\
\hline 2 & 0 & 5 & 36.1 & -30.6 & 9.4 & -9.5 \\
\hline 2 & 0 & 6 & 39.1 & -33.4 & 38.6 & -40.7 \\
\hline 2 & 0 & 7 & 13.8 & 16.7 & 3.0 & $-\quad 2.0$ \\
\hline 2 & 0 & 8 & 27.7 & 25.6 & 20.4 & 20.8 \\
\hline 2 & 0 & 9 & 27.0 & 27.3 & 20.8 & 18.4 \\
\hline 2 & 0 & 10 & 16.5 & 16.8 & 19.7 & 19.7 \\
\hline 2 & 0 & 11 & 16.1 & 16.2 & 12.1 & 11.4 \\
\hline 2 & 0 & 12 & 44.3 & 37.6 & & \\
\hline 2 & 0 & 13 & 4.4 & 6.5 & & \\
\hline 2 & 1 & 0 & 10.7 & 9.8 & & \\
\hline 2 & 1 & 1 & 63.7 & 74.4 & & \\
\hline 2 & 1 & 2 & 76.4 & 91.4 & & \\
\hline 2 & 1 & 3 & 57.7 & 66.0 & 36.4 & 37.9 \\
\hline 2 & 1 & 4 & 41.3 & 35.1 & 21.3 & 21.0 \\
\hline 2 & 1 & 5 & 32.7 & 30.9 & 34.7 & 32.9 \\
\hline 2 & 1 & 6 & 26.0 & -21.4 & 12.3 & -11.9 \\
\hline 2 & 1 & 7 & 22.9 & 19.6 & 6.8 & 6.2 \\
\hline 2 & 1 & 8 & 0.0 & $\begin{array}{l}-\quad 0.6 \\
\end{array}$ & 0.0 & 0.6 \\
\hline 2 & 1 & 9 & 4.2 & $\begin{array}{l}-\quad 3.7 \\
\end{array}$ & 0.0 & 3.0 \\
\hline 2 & 1 & 10 & 8.2 & 9.4 & 9.8 & 10.5 \\
\hline 2 & 1 & 11 & 0.0 & 3.0 & 14.7 & 15.0 \\
\hline 2 & 1 & 12 & 0.0 & 0.9 & 2.0 & 2.2 \\
\hline 2 & 1 & 13 & 13.2 & 11.9 & & \\
\hline 2 & 2 & 0 & 16.4 & -12.8 & 43.2 & -42.5 \\
\hline 2 & 2 & 1 & 29.1 & 33.3 & 14.8 & 12.6 \\
\hline 2 & 2 & 2 & 43.4 & 38.2 & 52.6 & 61.6 \\
\hline 2 & 2 & 3 & 35.3 & 27.4 & 27.7 & 27.1 \\
\hline 2 & 2 & 4 & 20.1 & 19.8 & 20.1 & 19.1 \\
\hline 2 & 2 & 5 & 48.5 & -44.1 & 24.1 & -20.8 \\
\hline 2 & 2 & 6 & 43.6 & 36.3 & 40.0 & 41.2 \\
\hline 2 & 2 & 7 & 16.5 & 15.6 & 3.1 & 2.2 \\
\hline 2 & 2 & 8 & 9.9 & 8.4 & 3.3 & 2.3 \\
\hline 2 & 2 & 9 & 13.2 & 11.1 & 7.3 & -7.0 \\
\hline 2 & 2 & 10 & 50.9 & -56.8 & 36.2 & -41.1 \\
\hline 2 & 2 & 11 & 21.9 & -21.5 & 8.3 & $-\quad 7.4$ \\
\hline 2 & 2 & 12 & 28.1 & -29.0 & 15.9 & -16.3 \\
\hline 2 & 2 & 13 & 4.0 & 4.8 & & \\
\hline 2 & 3 & 0 & 44.8 & 44.4 & 44.1 & 51.7 \\
\hline 2 & 3 & 1 & 21.1 & 17.4 & 27.9 & -27.3 \\
\hline 2 & 3 & 2 & 26.3 & 22.1 & 19.5 & 15.9 \\
\hline 2 & 3 & 3 & 55.0 & -56.9 & 49.2 & -55.5 \\
\hline 2 & 3 & 4 & 40.7 & 40.7 & 28.4 & 28.7 \\
\hline 2 & 3 & 5 & 64.9 & -72.0 & 37.7 & -43.5 \\
\hline 2 & 3 & 6 & 13.4 & -11.1 & 13.1 & 11.7 \\
\hline 2 & 3 & 7 & 17.4 & 15.6 & 0.0 & 1.4 \\
\hline 2 & 3 & 8 & 11.6 & -10.3 & 0.0 & 0.1 \\
\hline 2 & 3 & 9 & 8.4 & 5.8 & 10.2 & $-\quad 6.9$ \\
\hline 2 & 3 & 10 & 12.7 & 12.5 & 9.4 & -7.0 \\
\hline 2 & 3 & 11 & 12.4 & -14.0 & 11.2 & -11.0 \\
\hline
\end{tabular}

\begin{tabular}{|c|c|c|c|c|c|c|}
\hline$h$ & $k$ & $l$ & $\left|F_{0}\right|$ & $F_{c}$ & $\left|F_{0}\right|$ & B \\
\hline 2 & 3 & 12 & 2.9 & $-\quad 2.4$ & & \\
\hline 2 & 4 & 0 & 14.9 & -14.1 & 18.5 & -18.1 \\
\hline 2 & 4 & 1 & 14.2 & 11.6 & 2.1 & \\
\hline 2 & 4 & 2 & 10.9 & $\begin{array}{r}-\quad 9.7 \\
-\end{array}$ & 0.0 & $\begin{array}{l}-1.8 \\
\end{array}$ \\
\hline$\overline{2}$ & 4 & 3 & 4.2 & 3.7 & 2.4 & 4.1 \\
\hline 2 & $\begin{array}{l}t \\
4\end{array}$ & 4 & 31.7 & -31.5 & $\begin{array}{l}32 .+ \\
32.9\end{array}$ & $\begin{array}{r}4.1 \\
-37.0\end{array}$ \\
\hline 2 & 4 & 5 & 27.5 & 23.9 & 33.5 & 32.0 \\
\hline 2 & 4 & 6 & 40.1 & -33.7 & 38.7 & -45.4 \\
\hline 2 & 4 & 7 & 15.0 & -11.4 & 0.0 & 2.5 \\
\hline 2 & 4 & 8 & 23.1 & 17.8 & 14.7 & 12.0 \\
\hline 2 & 4 & 9 & 20.1 & -20.7 & 0.0 & 2.5 \\
\hline 2 & 4 & 10 & 37.0 & 36.3 & 15.6 & 19.8 \\
\hline$\overline{2}$ & 4 & 11 & 4.8 & $-\quad 7.4$ & 7.3 & $\begin{array}{l}-\quad 6.2 \\
\end{array}$ \\
\hline 2 & 4 & 12 & 25.9 & 30.8 & & \\
\hline 2 & 5 & 0 & 107.6 & -116.1 & 60.1 & -65.2 \\
\hline 2 & 5 & 1 & 78.6 & 85.2 & 60.9 & 63.8 \\
\hline 2 & 5 & 2 & 20.0 & -13.4 & 17.7 & $\begin{array}{r}-13.0 \\
-13.6\end{array}$ \\
\hline$\overline{2}$ & 5 & 3 & 47.8 & 45.3 & 33.5 & 32.9 \\
\hline 2 & 5 & 4 & 3.5 & 3.8 & 26.8 & -23.2 \\
\hline 2 & 5 & 5 & 0.0 & 1.6 & 33.0 & 32.2 \\
\hline 2 & 5 & 6 & 33.6 & -30.7 & 22.5 & -22.1 \\
\hline 2 & 5 & 7 & 28.5 & 28.5 & 14.7 & 12.3 \\
\hline$\overline{2}$ & 5 & 8 & 0.0 & 2.2 & 0.0 & 2.0 \\
\hline 2 & 5 & 9 & 8.1 & -6.0 & 3.1 & $\begin{array}{r}-2.0 \\
-\quad 2.6\end{array}$ \\
\hline 2 & 5 & 10 & 15.8 & 15.8 & 8.4 & 8.7 \\
\hline 2 & 5 & 11 & -0.0 & 0.4 & 16.9 & 14.9 \\
\hline 2 & 5 & 12 & 6.3 & $\begin{array}{l}-\quad 9.0 \\
\end{array}$ & & \\
\hline 2 & 6 & 0 & 21.2 & -18.8 & 25.4 & -24.1 \\
\hline 2 & 6 & 1 & 23.9 & -21.2 & 0.0 & $\begin{array}{r}-\quad 0.9 \\
-\end{array}$ \\
\hline 2 & 6 & 2 & 25.2 & 21.8 & 35.2 & 34.7 \\
\hline 2 & 6 & 3 & 0.0 & 0.7 & 13.0 & 11.7 \\
\hline 2 & 6 & 4 & 44.0 & 42.3 & 30.4 & 26.9 \\
\hline$\overline{2}$ & 6 & 5 & 0.0 & 3.0 & 10.0 & $\begin{array}{l}-\quad 9.2 \\
\end{array}$ \\
\hline 2 & 6 & 6 & 16.9 & 16.0 & 29.2 & 28.9 \\
\hline 2 & 6 & 7 & 0.0 & 0.7 & 5.7 & $-\quad 4.4$ \\
\hline 2 & 6 & 8 & 10.1 & -11.0 & 0.0 & $\begin{array}{l}7.7 \\
0.0\end{array}$ \\
\hline 2 & 6 & 9 & 0.0 & $\begin{array}{r}1.0 \\
2.0\end{array}$ & 6.7 & $\begin{array}{l}0.0 \\
-\quad 6.1\end{array}$ \\
\hline 2 & 6 & 10 & 24.6 & -27.6 & 30.0 & -28.6 \\
\hline 2 & 7 & 0 & 0.0 & $-\quad 3.0$ & 16.2 & -15.8 \\
\hline 2 & 7 & 1 & 5.2 & $-\quad 2.7$ & 16.4 & -14.0 \\
\hline 2 & 7 & 2 & 18.8 & -21.7 & 28.9 & -28.5 \\
\hline 2 & 7 & 3 & 52.0 & -50.9 & 39.5 & -43.4 \\
\hline 2 & 7 & 4 & 4.0 & 2.6 & 3.2 & $\begin{array}{r}-2.4 \\
-\quad 2.5\end{array}$ \\
\hline 2 & 7 & 5 & 49.3 & -46.7 & 31.1 & -25.6 \\
\hline 2 & 7 & 6 & 0.0 & 1.5 & 10.4 & 8.8 \\
\hline 2 & 7 & 7 & 5.9 & 6.9 & 0.0 & 1.9 \\
\hline 2 & 7 & 8 & 5.6 & $-\quad 3.7$ & 0.0 & 1.5 \\
\hline 2 & 7 & 9 & 0.0 & $-\quad 1.5$ & 11.4 & -9.2 \\
\hline$\overline{2}$ & 7 & 10 & 0.0 & 2.8 & 8.8 & -7.2 \\
\hline 2 & 8 & 0 & 9.7 & $\begin{array}{l}-2.0 \\
-\end{array}$ & $\begin{array}{r}0.0 \\
12.4\end{array}$ & -10.0 \\
\hline 2 & 8 & 1 & $\begin{array}{r}8.1 \\
26.2\end{array}$ & $=26.5$ & 17.5 & -15.7 \\
\hline 2 & 8 & 2 & 16.2 & -14.6 & 14.4 & -13.5 \\
\hline 2 & 8 & 3 & 20.1 & -21.8 & 25.3 & -22.9 \\
\hline 2 & 8 & 4 & 16.6 & -15.1 & 27.5 & -24.5 \\
\hline 2 & 8 & 5 & 25.7 & 25.0 & 3.3 & 2.9 \\
\hline 2 & 8 & 6 & 35.0 & -33.8 & 33.2 & -30.9 \\
\hline 2 & 8 & 7 & 5.6 & $-\quad 7.4$ & 3.0 & -3.1 \\
\hline 2 & 8 & 8 & $\begin{array}{l}2.0 \\
2.3\end{array}$ & 2.9 & $\begin{array}{r}0.0 \\
11.7\end{array}$ & 8.8 \\
\hline 2 & 8 & 9 & 0.0 & $-\quad 0.8$ & 11.7 & 9.6 \\
\hline 2 & 8 & 10 & 45.0 & 41.8 & & \\
\hline 2 & 9 & 0 & 22.8 & 23.3 & 25.5 & 25.7 \\
\hline 2 & 9 & 1 & 27.3 & 27.7 & 32.9 & 33.6 \\
\hline 2 & 9 & 2 & 30.7 & 30.3 & 32.9 & $\begin{array}{l}32.0 \\
32.0\end{array}$ \\
\hline 2 & 9 & 3 & 36.1 & 36.8 & 23.1 & 20.7 \\
\hline 2 & 9 & 4 & 8.3 & 6.6 & 3.2 & 4.8 \\
\hline 2 & 9 & 5 & 29.9 & 31.7 & 25.9 & 24.2 \\
\hline 2 & 9 & 6 & 0.0 & 0.3 & 2.9 & -3.4 \\
\hline 2 & 9 & 7 & 10.2 & 10.4 & 2.6 & 5.0 \\
\hline 2 & 9 & 8 & 0.0 & 0.8 & & \\
\hline 2 & 9 & 9 & 9.0 & $-\quad 9.3$ & & \\
\hline 2 & 10 & 0 & 0.0 & 0.4 & 0.0 & -2.1 \\
\hline 2 & 10 & 1 & 8.2 & -8.3 & 3.2 & 5.2 \\
\hline 2 & 10 & 2 & 8.2 & 7.7 & 17.2 & 14.9 \\
\hline 2 & 10 & 3 & 5.7 & 7.2 & 13.7 & 12.0 \\
\hline 2 & 10 & 4 & 30.3 & 31.1 & 20.8 & 21.8 \\
\hline 2 & 10 & 5 & 7.5 & 5.3 & 2.8 & $\begin{array}{r}-3.0 \\
\end{array}$ \\
\hline 2 & 10 & 6 & 24.7 & 21.1 & 23.4 & 22.7 \\
\hline 2 & 10 & 7 & 4.4 & $-\quad 3.9$ & 0.0 & -1.7 \\
\hline 2 & 11 & 0 & 20.9 & 25.0 & 6.4 & 6.9 \\
\hline 2 & 11 & 1 & 24.7 & -27.8 & 12.7 & -12.8 \\
\hline 2 & 11 & 2 & 11.8 & -11.0 & 2.8 & -4.8 \\
\hline 2 & 11 & 3 & 37.0 & -36.0 & 35.7 & -31.5 \\
\hline 2 & 11 & 4 & 10.9 & $\begin{array}{r}\quad 9.0 \\
-\end{array}$ & 2.5 & 3.5 \\
\hline 2 & 11 & 5 & 11.9 & -14.4 & 18.4 & -16.9 \\
\hline 2 & 11 & 6 & 12.6 & 13.5 & 8.0 & 8.7 \\
\hline 2 & 11 & 7 & 7.8 & $-\quad 7.5$ & & \\
\hline 2 & 12 & 0 & 6.5 & 8.3 & 5.1 & 5.8 \\
\hline
\end{tabular}

Table 2. 


\begin{tabular}{|c|c|c|c|c|c|c|}
\hline$h$ & $k$ & $l$ & $\left|F_{0}\right|$ & $F c$ & {$\left[F_{0}\right]$} & $\boldsymbol{F}_{c}$ \\
\hline 2 & 12 & 1 & 0.0 & $-\quad 0.6$ & 0.0 & -0.2 \\
\hline 2 & 12 & 2 & 15.6 & -15.5 & 11.9 & -11.9 \\
\hline 2 & 12 & 3 & 0.0 & $\begin{array}{l}-\quad 0.7 \\
-\end{array}$ & 0.0 & $\begin{array}{l}-1.9 \\
-1.0\end{array}$ \\
\hline 2 & 12 & 4 & 17.1 & -18.9 & 18.7 & -17.7 \\
\hline 2 & 12 & 5 & 7.3 & 8.1 & & \\
\hline 2 & 13 & 0 & 13.6 & -13.8 & & \\
\hline 2 & 13 & 1 & 4.5 & 5.8 & & \\
\hline 2 & 13 & 2 & 9.4 & $\begin{array}{l}-\quad 9.7 \\
\end{array}$ & & \\
\hline 2 & 13 & 3 & 21.5 & 25.8 & & \\
\hline 3 & 0 & 1 & 22.1 & -22.8 & 14.0 & -18.9 \\
\hline 3 & 0 & 3 & 52.4 & -48.8 & 19.7 & -16.4 \\
\hline 3 & 0 & 4 & 48.6 & -50.2 & 23.2 & -24.2 \\
\hline 3 & 0 & 5 & 20.2 & 17.3 & 8.4 & 1.8 \\
\hline 3 & 0 & 6 & 26.4 & -26.2 & 13.2 & -11.0 \\
\hline 3 & 0 & 7 & 13.1 & 8.4 & 3.2 & 1.7 \\
\hline 3 & 0 & 8 & 16.4 & 19.2 & 23.6 & -26.4 \\
\hline $\begin{array}{l}0 \\
3\end{array}$ & 0 & 9 & $\begin{array}{l}0.4 .8 \\
28.8\end{array}$ & -32.6 & 30.6 & $\begin{array}{r}20 . \\
-36.0\end{array}$ \\
\hline 3 & 0 & 10 & 27.5 & -32.1 & 20.7 & $\begin{array}{r}-24.9 \\
-\quad 20\end{array}$ \\
\hline 3 & 0 & 11 & 23.7 & -24.7 & 21.4 & - 19.4 \\
\hline 3 & 0 & 12 & 3.0 & $-\quad 4.6$ & & \\
\hline 3 & 1 & 2 & 25.0 & -27.0 & & \\
\hline 3 & 1 & 3 & 31.5 & -25.6 & 23.1 & -20.8 \\
\hline $\begin{array}{l}3 \\
3\end{array}$ & 1 & 4 & 0.0 & $\begin{array}{r}-2.4 \\
-\quad 1\end{array}$ & 16.7 & 13.8 \\
\hline $\begin{array}{l}0 \\
3 \\
3\end{array}$ & 1 & $\begin{array}{l}4 \\
5\end{array}$ & 29.2 & 26.9 & 25.5 & 24.1 \\
\hline 3 & 1 & 6 & 31.2 & 23.2 & 23.1 & 21.5 \\
\hline 3 & 1 & 7 & 5.9 & 6.7 & 12.4 & 10.1 \\
\hline 3 & 1 & 8 & 28.6 & 24.5 & 24.5 & 24.0 \\
\hline 3 & 1 & 9 & 32.7 & 32.2 & 19.0 & 17.1 \\
\hline 3 & 1 & 10 & 17.9 & 18.4 & 11.3 & 8.6 \\
\hline $\begin{array}{l}0 \\
3 \\
3\end{array}$ & 1 & 11 & 24.1 & 23.4 & 3.3 & $\begin{array}{l}0.0 \\
6.0\end{array}$ \\
\hline 3 & 1 & 12 & 5.8 & $-\quad 7.6$ & & \\
\hline 3 & 2 & 1 & 29.5 & 30.6 & 26.7 & 22.5 \\
\hline $\begin{array}{l}3 \\
3\end{array}$ & 2 & 3 & 0.0 & 1.1 & 11.9 & 10.7 \\
\hline 3 & 2 & 4 & 27.5 & -24.5 & 17.5 & -17.4 \\
\hline 3 & 2 & 5 & 37.5 & -29.1 & 26.3 & -25.3 \\
\hline 3 & 2 & 6 & 43.0 & 37.7 & 49.0 & 50.9 \\
\hline $\begin{array}{l}3 \\
3\end{array}$ & 2 & 7 & $\begin{array}{l}40.0 \\
13.4\end{array}$ & $\begin{array}{l}-14.1 \\
\end{array}$ & 17.7 & $\begin{array}{r}-15.2 \\
-15\end{array}$ \\
\hline 3 & 2 & 8 & 28.7 & 31.6 & 20.9 & 21.6 \\
\hline 3 & 2 & 9 & 12.5 & 12.0 & 17.4 & 16.9 \\
\hline 3 & 2 & 10 & 10.2 & 8.5 & 22.0 & 23.6 \\
\hline 3 & 2 & 11 & 19.9 & 19.8 & 12.4 & 10.9 \\
\hline 3 & 2 & 12 & 0.0 & $-\quad 0.8$ & & \\
\hline $\begin{array}{l}0 \\
3 \\
\end{array}$ & 3 & 1 & 7.3 & 8.8 & & \\
\hline $\begin{array}{l}3 \\
3\end{array}$ & 3 & 2 & 58.1 & 60.2 & & \\
\hline 3 & 3 & 3 & 63.9 & 64.1 & 39.9 & 36.5 \\
\hline 3 & 3 & 4 & 23.6 & 24.6 & 16.7 & $\begin{array}{l}0.00 \\
14.2\end{array}$ \\
\hline 3 & 3 & 5 & 20.1 & 16.8 & 9.2 & 7.8 \\
\hline 3 & 3 & 6 & 8.1 & $-\quad 9.2$ & 0.0 & 0.6 \\
\hline 3 & 3 & 7 & 21.6 & -19.6 & 0.0 & $\begin{array}{l}0.2 \\
-\quad 0.2\end{array}$ \\
\hline $\begin{array}{l}0 \\
3\end{array}$ & $\begin{array}{l}5 \\
3\end{array}$ & 8 & 6.3 & $\begin{array}{r}18.0 \\
-\quad 4.9\end{array}$ & $\begin{array}{r}18.0 \\
18.0\end{array}$ & $\begin{array}{r}-15.5 \\
-\end{array}$ \\
\hline 3 & 3 & 9 & 0.0 & 1.0 & 9.9 & 7.7 \\
\hline 3 & 3 & 10 & 15.2 & -14.1 & $\begin{array}{r}12.0 \\
12.2\end{array}$ & -11.4 \\
\hline 3 & 3 & 11 & 0.0 & $-\quad 1.5$ & & \\
\hline 3 & 3 & 12 & 6.2 & 5.5 & & \\
\hline 3 & 4 & 1 & 12.8 & -10.8 & 27.6 & -25.8 \\
\hline 3 & 4 & 2 & 27.5 & -23.1 & 0.0 & \\
\hline 3 & $\begin{array}{l}4 \\
4\end{array}$ & 3 & 6.8 & $\begin{array}{r}20.1 \\
3.8\end{array}$ & $\begin{array}{l}4.0 \\
4.5\end{array}$ & $\begin{array}{l}0.5 \\
2.5\end{array}$ \\
\hline 3 & 4 & 4 & 50.4 & -53.3 & 35.1 & -34.6 \\
\hline 3 & 4 & 5 & 53.6 & 52.7 & 35.1 & 36.1 \\
\hline 3 & 4 & 6 & 49.0 & -46.1 & 34.2 & -30.3 \\
\hline 3 & 4 & 7 & 34.7 & 36.5 & 36.7 & 38.0 \\
\hline 3 & 4 & 8 & 16.7 & -16.6 & 32.4 & $\begin{array}{r}0.0 .1 \\
-36.1\end{array}$ \\
\hline 3 & 4 & 9 & 4.3 & $\begin{array}{l}-\quad 3.3 \\
\end{array}$ & 0.0 & $\begin{array}{l}-\quad 1.6 \\
\end{array}$ \\
\hline 3 & 4 & 10 & 16.5 & -18.9 & 18.1 & -20.9 \\
\hline 3 & 4 & 11 & 11.2 & -14.0 & & \\
\hline
\end{tabular}

\begin{tabular}{|c|c|c|c|c|c|c|}
\hline \multirow[b]{2}{*}{$h$} & \multirow[b]{2}{*}{$k$} & \multirow[b]{2}{*}{$l$} & \multicolumn{2}{|c|}{ A } & \multicolumn{2}{|c|}{ B } \\
\hline & & & $\left|F_{0}\right|$ & $F_{c}$ & $\left|F_{0}\right|$ & $F_{c}$ \\
\hline 3 & 5 & 1 & 35.7 & -35.1 & 44.2 & -41.9 \\
\hline 3 & 5 & 2 & 42.6 & -40.9 & 44.1 & -41.4 \\
\hline 3 & 5 & 3 & 24.2 & -25.8 & 36.3 & -38.0 \\
\hline 3 & 5 & 4 & 39.9 & -34.5 & 28.8 & -22.7 \\
\hline 3 & 5 & 5 & 0.0 & 1.8 & 3.2 & \\
\hline 3 & 5 & 6 & 6.2 & $-\quad 5.6$ & 0.0 & $-\quad 0.6$ \\
\hline 3 & 5 & 7 & 37.0 & -37.9 & 20.8 & -19.2 \\
\hline 3 & 5 & 8 & 13.9 & 14.3 & 17.2 & 14.5 \\
\hline 3 & 5 & 9 & 15.5 & 13.7 & 0.0 & 1.2 \\
\hline 3 & 5 & 10 & 10.3 & 11.9 & 10.4 & 9.9 \\
\hline 3 & 5 & 11 & 21.2 & 23.2 & & \\
\hline 3 & 6 & 1 & 13.8 & 11.2 & 18.6 & 17.3 \\
\hline 3 & 6 & 2 & 5.5 & $-\quad 6.7$ & 0.0 & 0.8 \\
\hline 3 & 6 & 3 & 11.5 & 8.3 & 9.8 & 5.8 \\
\hline 3 & 6 & 4 & 43.8 & 41.7 & 3.2 & 6.3 \\
\hline 3 & 6 & 5 & 37.0 & -36.1 & 25.4 & -25.8 \\
\hline 3 & 6 & 6 & 44.9 & 44.9 & 39.1 & 39.8 \\
\hline $\begin{array}{l}0 \\
3\end{array}$ & 6 & 7 & 26.6 & $\begin{array}{l}-27.5 \\
-\end{array}$ & $\begin{array}{l}20.3 \\
20.3\end{array}$ & $\begin{array}{r}00.0 \\
-19.8\end{array}$ \\
\hline 3 & 6 & 8 & $\begin{array}{r}20.0 \\
0.0\end{array}$ & 0.6 & $\begin{array}{l}16.3 \\
16.3\end{array}$ & $\begin{array}{l}19.0 \\
17.2\end{array}$ \\
\hline 3 & 6 & 9 & 11.0 & 11.1 & 11.5 & 11.1 \\
\hline 3 & 6 & 10 & 20.0 & 17.7 & 25.2 & 22.2 \\
\hline 3 & 6 & 11 & 13.0 & 16.3 & & \\
\hline 3 & 7 & 1 & 27.0 & 24.3 & 36.1 & 36.3 \\
\hline 3 & 7 & 2 & 31.1 & 29.7 & 22.7 & 23.6 \\
\hline $\begin{array}{l}0 \\
3\end{array}$ & 7 & 3 & 25.5 & 24.1 & 12.6 & 9.0 \\
\hline 3 & 7 & 4 & 12.5 & 10.7 & 3.3 & -3.2 \\
\hline $\begin{array}{l}3 \\
3\end{array}$ & 7 & 5 & 8.9 & 4.7 & 0.0 & 0.9 \\
\hline 3 & 7 & 6 & 14.0 & -14.8 & 12.4 & -10.8 \\
\hline 3 & 7 & 7 & 18.2 & -18.4 & 13.5 & -14.2 \\
\hline 3 & 7 & 8 & 12.6 & -13.2 & 14.7 & -18.3 \\
\hline 3 & 7 & 9 & 17.1 & -15.6 & 4.9 & -4.7 \\
\hline 3 & 7 & 10 & 15.2 & -13.0 & & \\
\hline 3 & 8 & 1 & 27.1 & -26.0 & 14.7 & -15.7 \\
\hline 3 & 8 & 2 & 30.7 & -32.3 & 3.3 & 3.7 \\
\hline 3 & 8 & 3 & 4.5 & -4.3 & 3.3 & -4.5 \\
\hline 3 & 8 & 4 & 15.4 & 18.0 & 0.0 & -0.3 \\
\hline 3 & 8 & 5 & 12.4 & 13.4 & 7.1 & 7.7 \\
\hline 3 & 8 & 6 & 13.4 & -15.2 & 16.4 & -16.3 \\
\hline . & 8 & 7 & $\begin{array}{r}1.4 . \\
4.0\end{array}$ & 1.8 & $\begin{array}{r}0.7 \\
0.0\end{array}$ & 1.9 \\
\hline $\begin{array}{l}3 \\
3\end{array}$ & 8 & 8 & $\begin{array}{r}4.0 \\
24.7\end{array}$ & $\begin{array}{l}1.0 \\
-24.9\end{array}$ & 22.8 & -22.9 \\
\hline 3 & 8 & 9 & 14.8 & -14.4 & 17.6 & -21.1 \\
\hline 3 & 8 & 10 & 8.9 & -8.7 & & \\
\hline 3 & 9 & 1 & 15.4 & -14.9 & 17.8 & -20.3 \\
\hline 3 & 9 & 2 & 19.3 & -22.1 & 14.5 & -15.3 \\
\hline $\begin{array}{l}\overrightarrow{3} \\
3\end{array}$ & 9 & 3 & 28.5 & -33.4 & 22.4 & -23.8 \\
\hline $\begin{array}{l}3 \\
3\end{array}$ & 9 & 4 & $\begin{array}{r}2.03 \\
0.0\end{array}$ & $\begin{array}{r}00.4 \\
-\quad 1.3\end{array}$ & 0.0 & 3.1 \\
\hline 3 & 9 & 5 & 15.5 & 16.9 & 20.5 & 20.9 \\
\hline 3 & 9 & 6 & 16.0 & 15.9 & 11.8 & 15.0 \\
\hline 3 & 9 & 7 & 14.9 & 16.7 & 0.0 & 4.9 \\
\hline 3 & 9 & 8 & 14.8 & 15.1 & 13.4 & 15.2 \\
\hline 3 & 9 & 9 & 15.1 & 14.5 & & \\
\hline $\begin{array}{l}3 \\
3 \\
3\end{array}$ & $\begin{array}{r}9 \\
10\end{array}$ & 1 & 12.0 & $\begin{array}{l}14.0 \\
14.4\end{array}$ & 13.5 & 14.5 \\
\hline 3 & 10 & 2 & 13.2 & -16.2 & 0.0 & -3.2 \\
\hline 3 & 10 & 3 & 8.2 & 12.5 & 2.8 & 4.2 \\
\hline 3 & 10 & 4 & 23.5 & 26.6 & 2.7 & 4.3 \\
\hline 3 & 10 & 5 & 15.6 & -17.1 & 10.9 & -11.6 \\
\hline $\begin{array}{l}3 \\
3\end{array}$ & 10 & 6 & 16.9 & 18.9 & 21.8 & 19.7 \\
\hline $\begin{array}{l}0 \\
3 \\
3\end{array}$ & 10 & 7 & 7.9 & -10.0 & & \\
\hline $\begin{array}{l}3 \\
3 \\
\end{array}$ & 11 & 1 & 20.9 & $\begin{array}{l}10.0 \\
26.6\end{array}$ & 25.6 & 27.2 \\
\hline $\begin{array}{l}3 \\
3\end{array}$ & 11 & 2 & 15.6 & 18.1 & 20.7 & 19.5 \\
\hline 3 & 11 & 3 & 0.0 & 4.8 & 2.3 & 5.8 \\
\hline 3 & 11 & 4 & 15.1 & 14.0 & 6.6 & 7.0 \\
\hline 3 & 12 & 1 & 7.3 & -10.8 & 9.8 & -13.1 \\
\hline 3 & 12 & 2 & 18.8 & -17.1 & & \\
\hline
\end{tabular}

Table 2.

factors calculated from the least squares refinements are given together with the coordinates of the hydrogen atoms calculated from the NMR experiments.

For the calculation of the atomic positions of hydrogen the ${ }^{1} \mathrm{H}$-NMR informations are not suitable without further assumptions from X-ray experiments about the hydrogen bridge bonds. A way out of this difficulty is the consideration of higher order interactions (second moments) in the NMR experiments ${ }^{8}$.
In the experiments regarded here, a two dimensional difference Fourier synthesis with the structure factors $F_{0}-F_{c}$ was performed. The projections of the difference in electron density (in arbitrary units) onto the $b c$ plane are shown in Fig. 3 and 4 for $\mathrm{Na}_{2} \mathrm{~S}_{2} \mathrm{O}_{6} \cdot 2 \mathrm{H}_{2} \mathrm{O}$ and $\mathrm{Li}_{2} \mathrm{~S}_{2} \mathrm{O}_{6} \cdot 2 \mathrm{H}_{2} \mathrm{O}$, respectively. From these projections one can see, that the hydro-

8 M. van Merrsche and J. M. Dereppe, J. Chim. Phys. 63, 17 [1966]. 


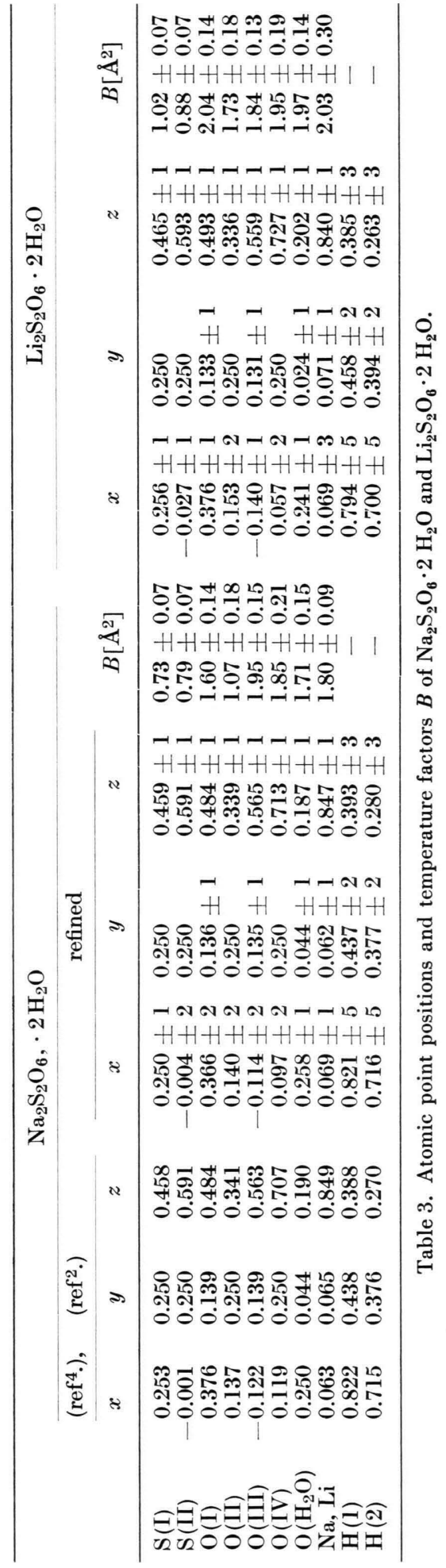

9 K. Nagauoro, M. Magoshes, and R. E. Rundle, J. Amer. Chem. Soc. 77, 6480 [1955]. gen bridge bonds are directed towards the oxygen atoms $\mathrm{O}$ (II) and $\mathrm{O}$ (III) (see Fig. 2 and Table 3 ). For the water oxygen $\mathrm{O}(\mathrm{V}, 2)$ (Fig. 2), the next nearest oxygen atoms are $\mathrm{O}(\mathrm{II}, 8)$ and $\mathrm{O}(\mathrm{III}, 7)$. Assuming in first approximation almost linear hydrogen bridge bonds between $\mathrm{O}\left(\mathrm{H}_{2} \mathrm{O}\right)$ and the next oxygen neighbours, agreement with the informations from the difference Fourier synthesis is found. The distances $\mathrm{O}(\mathrm{V}, 2) \ldots \mathrm{O}$ are: $\mathrm{O}(\mathrm{V}, 2) \ldots$ $\mathrm{O}(\mathrm{II}, 8)$ : $2.85 \AA$ and $2.77 \AA$; O (V, 2) ... O (III, 7) : $2.98 \AA$ and $2.96 \AA$ for $\mathrm{Na}_{2} \mathrm{~S}_{2} \mathrm{O}_{6} \cdot 2 \mathrm{H}_{2} \mathrm{O}$ and $\mathrm{Li}_{2} \mathrm{~S}_{2} \mathrm{O}_{6} \cdot$ $2 \mathrm{H}_{2} \mathrm{O}$, respectively. The bond angles $\mathrm{O} \ldots \mathrm{O}\left(\mathrm{H}_{2} \mathrm{O}\right)$ $\ldots \mathrm{O}$ are $109.4^{\circ}$ and $88.5^{\circ}$.

The infrared spectra of both compounds were studied in $\mathrm{KBr}$ and Nujol in the frequency region $4000 \mathrm{~cm}^{-1}$ to $100 \mathrm{~cm}^{-1}$. The spectra are illustrated in Fig. 5, 6 and 7. The assignment of the water specific bonds were accomplished by comparing the spectra of $\mathrm{Na}_{2} \mathrm{~S}_{2} \mathrm{O}_{6}, \mathrm{Na}_{2} \mathrm{~S}_{2} \mathrm{O}_{6} \cdot 2 \mathrm{H}_{2} \mathrm{O}, \mathrm{Na}_{2} \mathrm{~S}_{2} \mathrm{O}_{6} \cdot 2 \mathrm{D}_{2} \mathrm{O}$ and $\mathrm{Li}_{2} \mathrm{~S}_{2} \mathrm{O}_{6} \cdot 2 \mathrm{H}_{2} \mathrm{O}$. The symmetric $\mathrm{OH}$-stretching mode was found to be 3470 and $3400 \mathrm{~cm}^{-1}$ for the sodium and lithium compound, respectively. The symmetric $\mathrm{OH}$-stretching mode is dependent on the strength of the bridge bonds and therefore related to the $\mathrm{O}\left(\mathrm{H}_{2} \mathrm{O}\right) \ldots \mathrm{O}$ distances. NaKamoto et al. ${ }^{9}$ correlated the symmetric stretching mode to the $0 \ldots O$ distance. From the diagrams given in an illustration by Pimentel and McClellan ${ }^{10}$ we obtained for the $\mathrm{O} \ldots \mathrm{O}$ distances $\mathrm{O}(\mathrm{V}, 2)-\mathrm{O}(\mathrm{II}, 8)$ a value of 2.85 and $2.80 \AA$ which is in good agreement with the calculated value 2.85 and $2.77 \AA$.

\section{b) Determination of the Atomic Coordinates of Hydrogen}

The ${ }^{1} \mathrm{H}-\mathrm{NMR}$ experiments on crystal hydrates are useful to determine the direction cosines of the intramolecular $p-p$ lines of the different water molecules in the unit cell. Considering the dynamical behaviour of the water molecules the length of the $p-p$ vectors is available. No information about the position of the $p-p$ lines with respect to their water oxygens is gained from the proton magnetic resonance investigations, as the ${ }^{1} \mathrm{H}-\mathrm{NMR}$ experiments are invariant upon translational displacements of the $p-p$ lines. There are eight equivalent water molecules in the elementary cell. The $8 \mathrm{p}-\mathrm{p}$ lines belonging to them differ by the symmetry operations of the

10 G. C. Pimentel and A. L. McClellan, The Hydrogen Bond, W. H. Freeman \& Co., San Francisco/London 1960. 


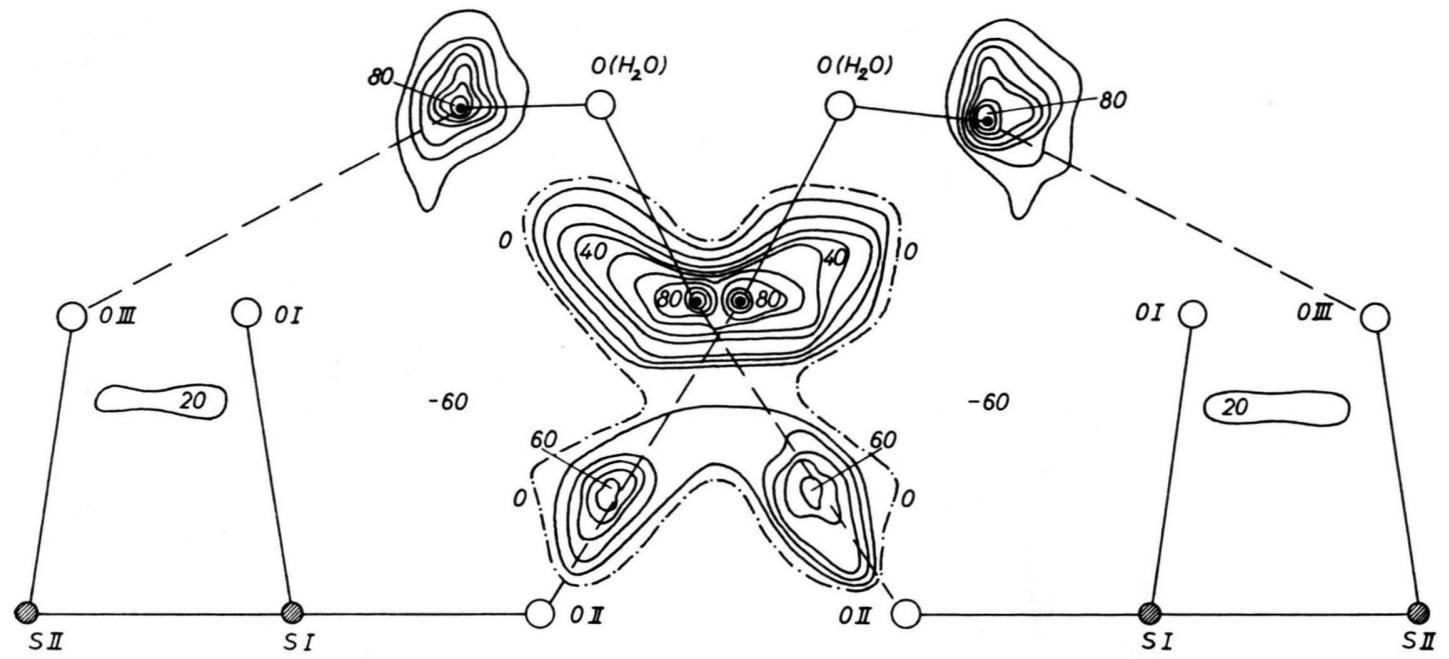

Fig. 3. Difference Fourier projection $(b c)$ of $\mathrm{Na}_{2} \mathrm{~S}_{2} \mathrm{O}_{6} \cdot 2 \mathrm{H}_{2} \mathrm{O}$ in arbitrary density units.

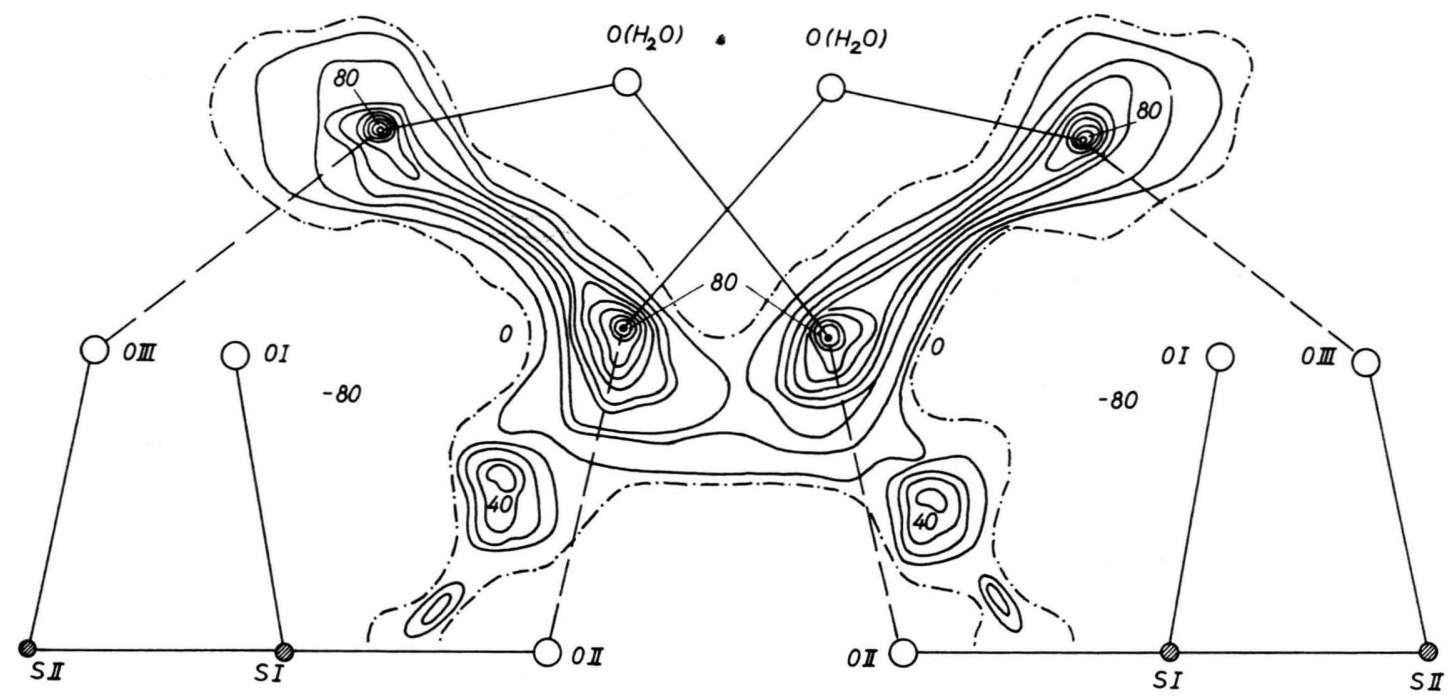

Fig. 4. Difference Fourier $(b c)$ projection of $\mathrm{Li}_{2} \mathrm{~S}_{2} \mathrm{O}_{6} \cdot 2 \mathrm{H}_{2} \mathrm{O}$ in arbitrary density units.

space group $\mathrm{D}_{2 \mathrm{~h}}^{16}-$ Pnma. For the determination of the hydrogen coordinates only one of these 8 vectors has to be fixed in the elementary cell. This fixation was performed by theoretical arguments. The method used is an analytical investigation of the triangle as shown in Fig. 8. In this triangle the $\mathrm{H}(1)-\mathrm{H}(2)$ bond length and direction cosines are known from NMR experiments. For the determination of the $H(1)$ and $H(2)$ coordinates the bond lengths and direction cosines of the $\mathrm{OH}$ bonds are necessary. The $\mathrm{OH}$-direction cosines were calculated under the assumption of equivalent bridge bonds for both hydrogen atoms and the restriction that the deviation from linear hydrogen bridge bonds (angles $\eta$ and $\lambda$ in Fig. 8) should be a minimum. If we denote the direction cosines of the line between two atoms $i, j(i, j=1-5)$ as $v_{i j}, \mu_{i j}, \omega_{i j}$ with respect to the crystal axes $a, b, c$ the following equations are valid:

$$
\begin{aligned}
\cos \varepsilon & =v_{12} \nu_{31}+\mu_{12} \mu_{31}+\omega_{12} \omega_{31} ; \\
\cos \eta & =v_{43} \nu_{31}+\mu_{43} \mu_{31}+\omega_{43} \omega_{31} ; \\
\cos \varepsilon^{\prime} & =v_{12} \nu_{32}+\mu_{12} \mu_{32}+\omega_{12} \omega_{32} ; \\
\cos \lambda & =v_{35} \nu_{32}+\mu_{35} \mu_{32}+\omega_{35} \omega_{32} ; \\
1 & =v_{31}{ }^{2}+\mu_{31}{ }^{2}+\omega_{31}{ }^{2} ; \\
1 & =v_{32}{ }^{2}+\mu_{32}{ }^{2}+\omega_{32}{ }^{2} .
\end{aligned}
$$




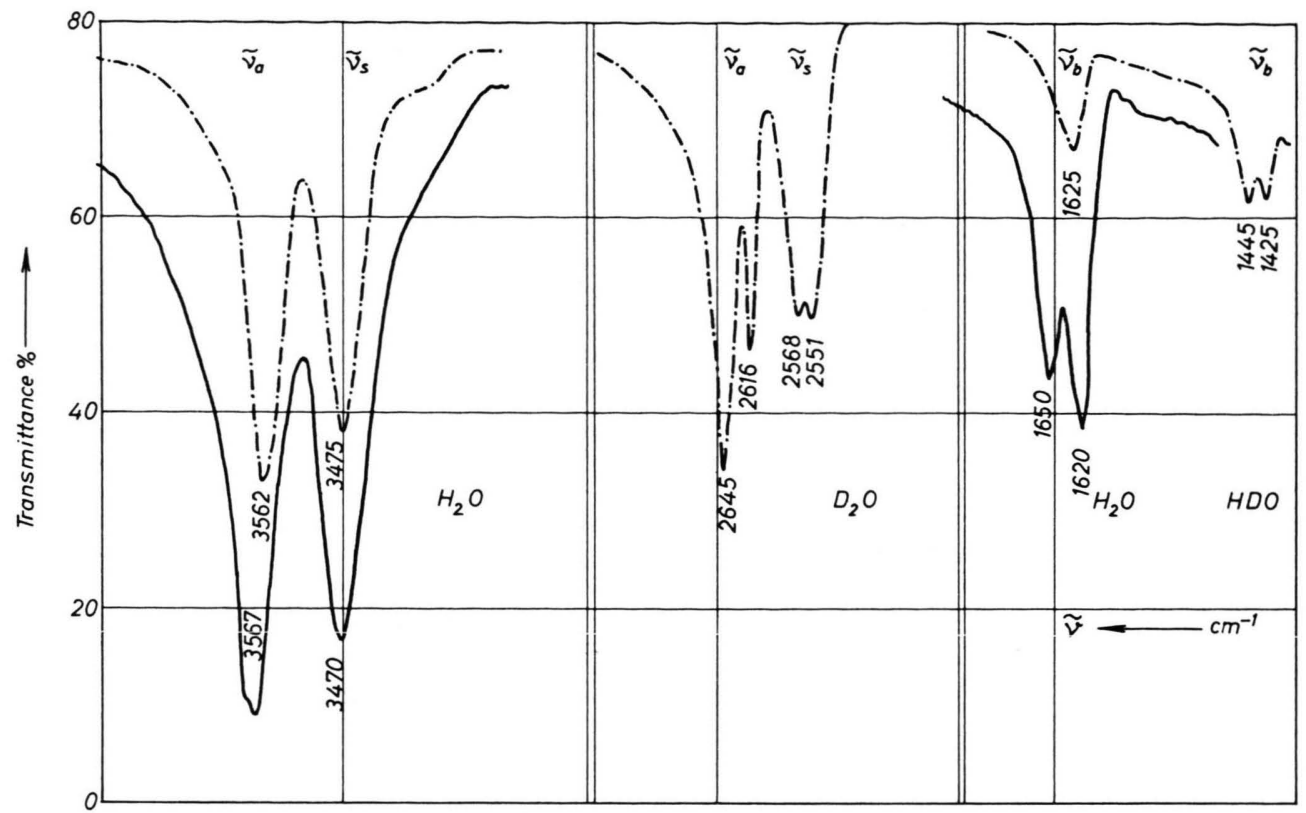

Fig. 5. The infrared spectra of $\mathrm{Na}_{2} \mathrm{~S}_{2} \mathrm{O}_{6} \cdot 2 \mathrm{H}_{2} \mathrm{O}(-)$ and $\mathrm{Na}_{2} \mathrm{~S}_{2} \mathrm{O}_{6} \cdot 2 \mathrm{D}_{2} \mathrm{O}(-\cdot-\cdot-)$ in the frequency region $4000 \mathrm{~cm}-1$ to $1500 \mathrm{~cm}^{-1}$.

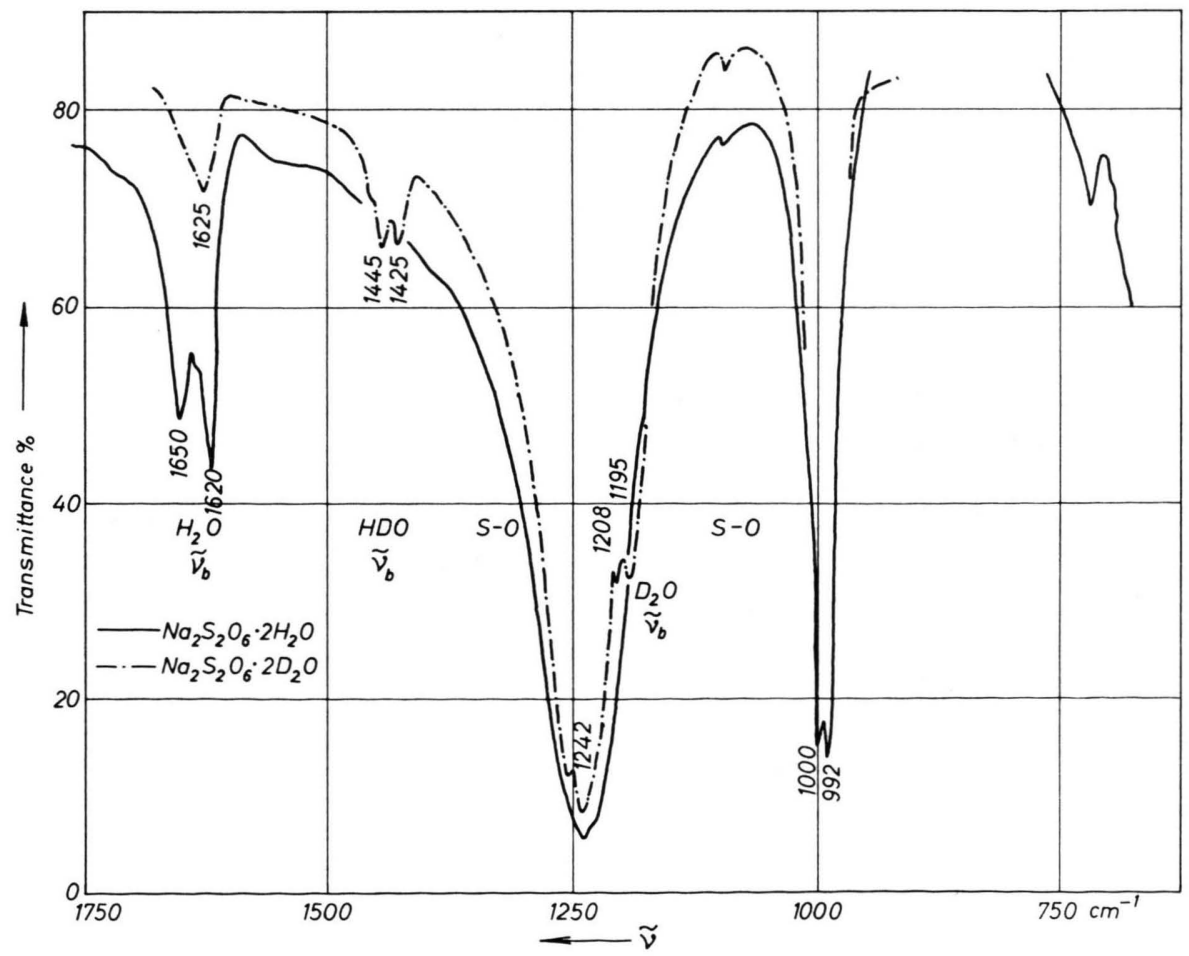

Fig. 6. The infrared spectra of $\mathrm{Na}_{2} \mathrm{~S}_{2} \mathrm{O}_{6} \cdot 2 \mathrm{H}_{2} \mathrm{O}$ and $\mathrm{Na}_{2} \mathrm{~S}_{2} \mathrm{O}_{6} \cdot 2 \mathrm{D}_{2} \mathrm{O}$ in the frequency region $1700 \mathrm{~cm}^{-1}-800 \mathrm{~cm}^{-1}$. 


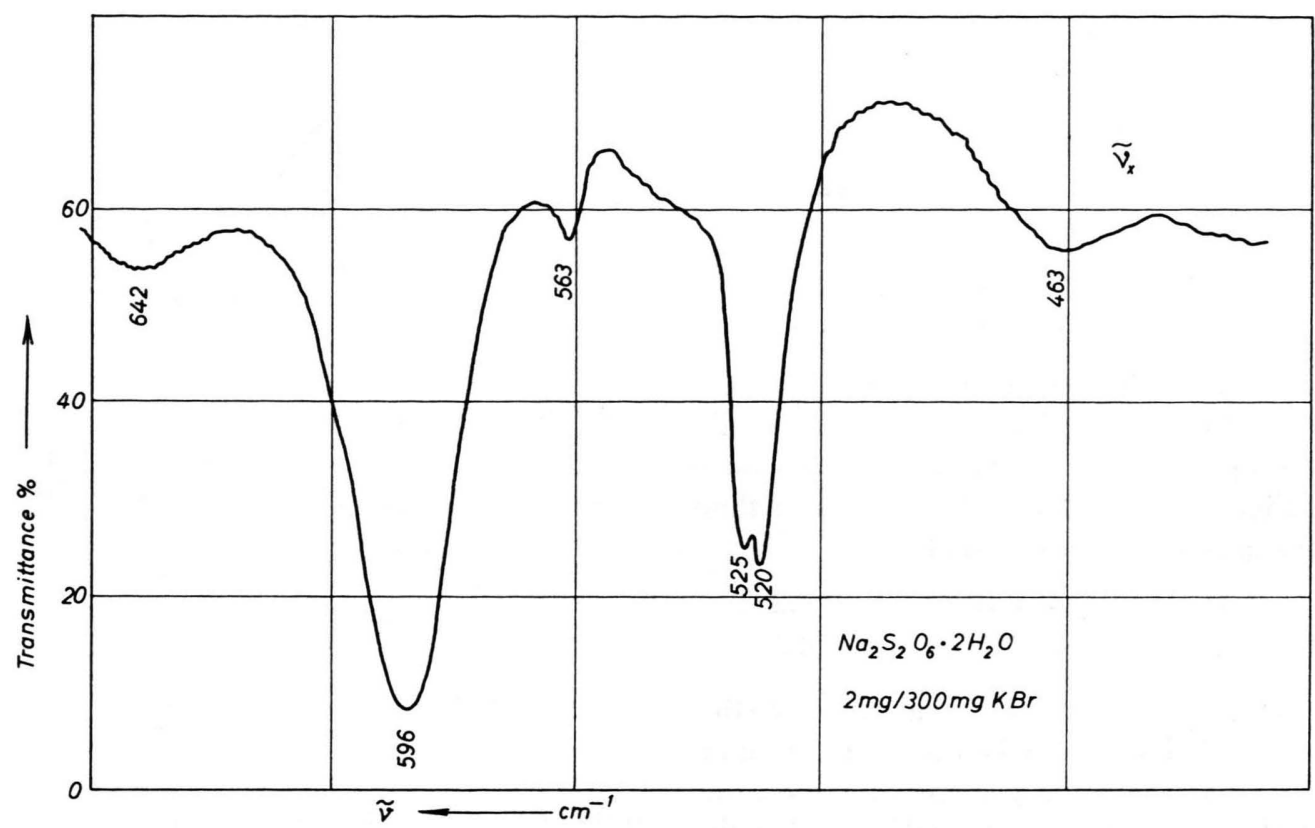

Fig. 7. The infrared spectrum of $\mathrm{Na}_{2} \mathrm{~S}_{2} \mathrm{O}_{6} \cdot 2 \mathrm{H}_{2} \mathrm{O}$ in the frequency region $650 \mathrm{~cm}^{-1}-400 \mathrm{~cm}^{-1}$.

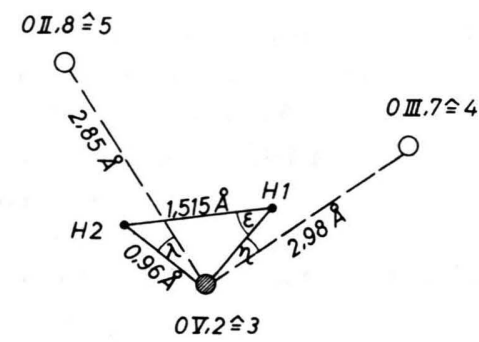

Fig. 8. Geometrical arrangement of a water molecule in its surrounding.

The direction cosines $v_{43}, \mu_{43}, \omega_{43}, v_{35}, \mu_{35}, \omega_{35}$ are directly available from the point positions of the corresponding oxygen atoms. The direction cosines $v_{12}, \mu_{12}, \omega_{12}$ are connected with the ${ }^{1} \mathrm{H}-\mathrm{NMR}$ experimental data by the transformation $v_{12} \equiv \sin \delta_{a}^{*}$ etc. (see paper I). The angles $\varepsilon$ and $\varepsilon^{\prime}$ may be determined from the $\mathrm{HH}$ - and $\mathrm{OH}$-bond lengths. Values of the $\mathrm{OH}$-bond lengths were obtained from the approximate linear relation

$$
R(\mathrm{OH})=1.574-0.2145 R(\mathrm{O} \ldots \mathrm{O})
$$

given by Hamilton ${ }^{11}$. With the known data $R(0 \ldots 0)$ from the structure determination we calculated for: $\mathrm{Na}_{2} \mathrm{~S}_{2} \mathrm{O}_{6} \cdot 2 \mathrm{H}_{2} \mathrm{O}$ :

$$
\begin{array}{ll}
R(\mathrm{O} \ldots \mathrm{O})=2.85 \AA, & R(\mathrm{OH})=0.96 \AA, \\
R(\mathrm{O} \ldots \mathrm{O})=2.98 \AA, & R(\mathrm{OH})=0.94 \AA ;
\end{array}
$$

11 W. C. Hamilton, Ann. Rev. Phys. Chem. 13, 28 [1962].
$\mathrm{Li}_{2} \mathrm{~S}_{2} \mathrm{O}_{6} \cdot 2 \mathrm{H}_{2} \mathrm{O}$ :

$$
\begin{array}{ll}
R(\mathrm{O} \ldots \mathrm{O})=2.77 \AA, & R(\mathrm{OH})=0.98 \AA, \\
R(\mathrm{O} \ldots \mathrm{O})=2.96 \AA, & R(\mathrm{OH})=0.94 \AA .
\end{array}
$$

From the relations given in Eq. (1) the unknown direction cosines of the $\mathrm{OH}$ bonds were eliminated and we received a functional relationship between the angles $\eta$ and $\lambda$. A plot of the function $\eta=f(\lambda)$ is given in Fig. 9 and Fig. 10, for $\mathrm{Na}_{2} \mathrm{~S}_{2} \mathrm{O}_{6} \cdot 2 \mathrm{H}_{2} \mathrm{O}$ and $\mathrm{Li}_{2} \mathrm{~S}_{2} \mathrm{O}_{6} \cdot 2 \mathrm{H}_{2} \mathrm{O}$, respectively. The smallest possible values for $\eta\left(\eta_{\min }\right)$ and $\lambda\left(\lambda_{\min }\right)$ are labelled; from these special points (Fig. 9 and 10) one can see, that no linear hydrogen bonds $(\eta, \lambda=0)$ are possible. Here the question arises what position of the water molecule between the two extremal arrangements $\left(\eta=\eta_{\min }\right.$ or $\left.\lambda=\lambda_{\min }\right)$ is the most probable one. The minimum of the potential field is certainly not given by one of the extremal positions. For each of the specified positions we may expect only the most stable location of one hydrogen bridge. Therefore we assume that the protons of the water molecule are situated in such a way, that the angles $\eta$ and $\lambda$ simultaneously show minimal deviation from the angles $\eta_{\min }$ and $\lambda_{\min }$. This assumption leads to the relation

$$
\left(\eta-\eta_{\min }\right)+\left(\lambda-\lambda_{\min }\right)=\min >0 .
$$


In addition the conditions

$$
\begin{array}{llll}
\lambda-\lambda_{\min }=\min & \text { for } & \lambda_{\min }>\eta_{\min } \\
\text { and } & \eta-\eta_{\min }=\min & \text { for } & \eta_{\min }>\lambda_{\min }
\end{array}
$$

were introduced. Connecting these two relations we obtain:

$$
\begin{array}{llll}
\eta+2 \lambda & =\min & \text { for } & \lambda_{\min }>\eta_{\min }, \\
\text { and } \quad \lambda+2 \eta=\min & \text { for } & \eta_{\min }>\lambda_{\min },
\end{array}
$$

respectively. Using the functional relation $\eta=f(\lambda)$ given in Fig. 9 and 10 a plot of $\eta+2 \lambda$ and $\lambda+2 \eta$ respectively as a function of $\lambda$ was possible and is shown in Fig. 9 and 10. From the minima of these curves the angles $\eta$ and $\lambda$ are available:

$$
\begin{array}{lll}
\mathrm{Na}_{2} \mathrm{~S}_{2} \mathrm{O}_{6} \cdot 2 \mathrm{H}_{2} \mathrm{O}: & \eta=10.8^{\circ}, & \lambda=13.2^{\circ} ; \\
\mathrm{Li}_{2} \mathrm{~S}_{2} \mathrm{O}_{6} \cdot 2 \mathrm{H}_{2} \mathrm{O}: & \eta=11.7^{\circ}, \quad \lambda=8.3^{\circ} .
\end{array}
$$

With the values of $\eta$ and $\lambda$ found the direction cosines of the $\mathrm{OH}$ bonds may be calculated from the set of Eq. (1). The hydrogen coordinates result from the $\mathrm{OH}$-direction cosines, the $\mathrm{OH}$-bond lengths, and the point position of the water oxygen. For the OH-bond length a mean value of $0.96 \AA$ was used. The results of the determination of the hydrogen positions are given in Table 3 .
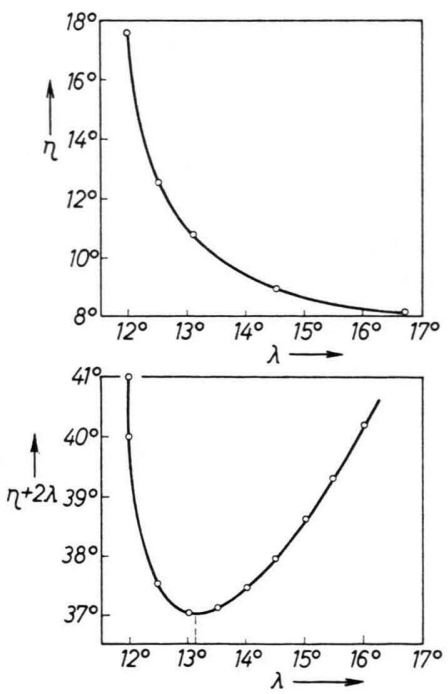

Fig. 9. The functions $\eta=f(\lambda)$ and $\eta+2 \lambda=f(\lambda)$ for $\mathrm{Na}_{2} \mathrm{~S}_{2} \mathrm{O}_{6} \cdot 2 \mathrm{H}_{2} \mathrm{O}$.

\section{Discussion of the Structure}

To sum up the results of the structure determination of $\mathrm{Na}_{2} \mathrm{~S}_{2} \mathrm{O}_{6} \cdot 2 \mathrm{H}_{2} \mathrm{O}$ and $\mathrm{Li}_{2} \mathrm{~S}_{2} \mathrm{O}_{6} \cdot 2 \mathrm{H}_{2} \mathrm{O}$ the atomic arrangements in both compounds shall be
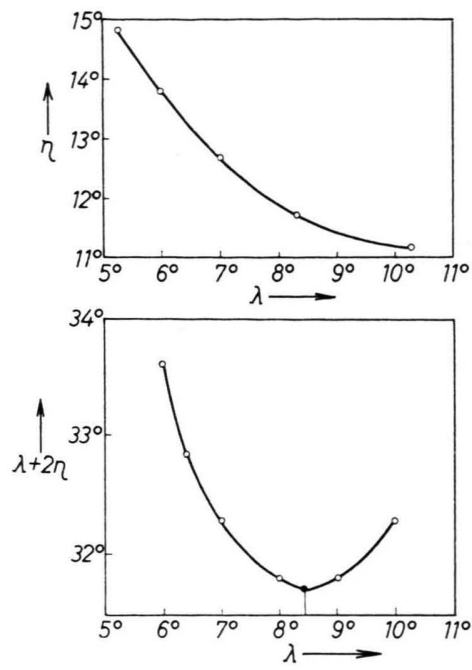

Fig. 10. The functions $\eta=f(\lambda)$ and $\lambda+2 \eta=f(\lambda)$ for $\mathrm{Li}_{2} \mathrm{~S}_{2} \mathrm{O}_{6} \cdot 2 \mathrm{H}_{2} \mathrm{O}$.

discussed. Each alkaline atom is surrounded octahedral by six oxygen atoms, two of them belonging to water molecules. These octahedrons are somewhat distorted. The SO-bond lengths of the $\mathrm{S}_{2} \mathrm{O}_{6}{ }^{2-}$ groups were found to be equivalent within the experimental accuracy $(1.45-1.46 \AA)$. The SSO and OSO angles

\begin{tabular}{|c|c|c|}
\hline Distances in $\AA$ : & A & B \\
\hline $\begin{array}{l}\text { S }(0,1)-O(0,1) \\
\text { S(I, 1)-O(II, }) \\
\text { S(II, 1)-O(III, 1) } \\
\text { S(II, 1)-O(IV,1) } \\
\text { S(I, 1)-S(II, 1) } \\
\text { H(1)-H (2) } \\
\text { O(V,2)-H(1) } \\
O(\mathrm{~V}, 2)-\mathrm{H}(2) \\
\mathrm{O}(\mathrm{V}, 2)-\mathrm{O}(\mathrm{III}, 7) \\
\mathrm{O}(\mathrm{V}, 2)-\mathrm{O}(\mathrm{II}, 8)\end{array}$ & $\begin{array}{l}1.458 \\
1.463 \\
1.450 \\
1.456 \\
2.15 \\
1.52 \\
0.96 \\
0.96 \\
2.98 \\
2.85\end{array}$ & $\begin{array}{l}1.447 \\
1.449 \\
1.457 \\
1.449 \\
2.14 \\
1.52 \\
0.96 \\
0.96 \\
2.96 \\
2.77\end{array}$ \\
\hline \multicolumn{3}{|l|}{ Angles: } \\
\hline $\begin{array}{l}\mathrm{H}(1)-\mathrm{O}(\mathrm{V}, 2)-\mathrm{H}(2) \\
\mathrm{O}(\mathrm{III}, 7)-\mathrm{O}(\mathrm{V}, 2)-\mathrm{H}(1) \\
\mathrm{O}(\mathrm{II}, 4)-\mathrm{O}(\mathrm{V}, 2)-\mathrm{H}(2) \\
\mathrm{S}(\mathrm{I}, 4)-\mathrm{O}(\mathrm{II}, 4)-\mathrm{H}(2) \\
\mathrm{S}(\mathrm{II}, 1)-\mathrm{O}(\mathrm{III}, 7)-\mathrm{H}(1) \\
\mathrm{S}(\mathrm{II}, 1)-\mathrm{S}(\mathrm{I}, 1)-\mathrm{O}(\mathrm{I}, 1) \\
\mathrm{O}(\mathrm{II}, 1)-\mathrm{S}(\mathrm{I}, 1)-\mathrm{S}(\mathrm{II}, 1) \\
\mathrm{S}(\mathrm{I}, 1)-\mathrm{S}(\mathrm{II}, 1)-\mathrm{O} \text { (III, 1) } \\
\mathrm{O}(\mathrm{IV}, 1)-\mathrm{S}(\mathrm{II}, 1)-\mathrm{S}(\mathrm{I}, 1)\end{array}$ & $\begin{aligned} 104.1^{\circ} \\
10.8^{\circ} \\
13.2^{\circ} \\
117.1^{\circ} \\
126.4^{\circ} \\
105.5^{\circ} \\
102.9^{\circ} \\
104.0^{\circ} \\
104.6^{\circ}\end{aligned}$ & $\begin{array}{r}104.5^{\circ} \\
11.7^{\circ} \\
8.3^{\circ} \\
115.5^{\circ} \\
133.3^{\circ} \\
106.1^{\circ} \\
102.1^{\circ} \\
105.9^{\circ} \\
107.1^{\circ}\end{array}$ \\
\hline
\end{tabular}
are between $103^{\circ}$ and $107^{\circ}$. Each hydrogen atom of the water molecules is coupled to an oxygen atom of a $\mathrm{S}_{2} \mathrm{O}_{6}{ }^{2-}$ group by a hydrogen bridge bond. The bridge bonds connect the $\mathrm{S}_{2} \mathrm{O}_{6}{ }^{2-}$ ions to chains along

Table 4. Interatomic distances and angles in $\mathrm{Na}_{2} \mathrm{~S}_{2} \mathrm{O}_{6} \cdot 2 \mathrm{H}_{2} \mathrm{O}$ (A) and $\mathrm{Li}_{2} \mathrm{~S}_{2} \mathrm{O}_{6} \cdot 2 \mathrm{H}_{2} \mathrm{O}$ (B). 
the crystallographic $a$ axis. Three oxygen atoms of one $\mathrm{S}_{2} \mathrm{O}_{6}{ }^{2-}$ ion participate in the bridge bonding. The oxygen atoms $\mathrm{O}$ (II) (see Fig. 2) share two hydrogen bonds. The shortest interatomic distance between two hydrogen atoms of two different water molecules was found to be 2.73 and $3.01 \AA$ for $\mathrm{Na}_{2} \mathrm{~S}_{2} \mathrm{O}_{6} \cdot 2 \mathrm{H}_{2} \mathrm{O}$ and $\mathrm{Li}_{2} \mathrm{~S}_{2} \mathrm{O}_{6} \cdot 2 \mathrm{H}_{2} \mathrm{O}$, respectively. In Table 4 a list of some interatomic distances and bond angles is given. The numbering of the different atoms may be seen in Fig. 2.

Our determination of the hydrogen positions makes the deviation of the bridge bonds from linearity as small as possible. The method chosen is based on the assumption that both hydrogen bridge bonds are energetically equivalent and that the potential energy of the water molecule reaches the minimum when the deviation angles $\eta$ and $\lambda$ are smallest. In this case the influence of the lone pair orbitals of the water molecules is not considered. The lone pair orbitals of the water molecules in $\mathrm{Na}_{2} \mathrm{~S}_{2} \mathrm{O}_{6} \cdot 2 \mathrm{H}_{2} \mathrm{O}$ and $\mathrm{Li}_{2} \mathrm{~S}_{2} \mathrm{O}_{6} \cdot 2 \mathrm{H}_{2} \mathrm{O}$ are directed approximately towards the alkaline ions and may influence the equilibrium positions of the water molecules. Another way in getting informations on the hydrogen positions would be a calculation of the minimum of the potential energy of the water molecule. Approximate calculations on this subject using a point charge model for the determination of the energy function were done by $\mathrm{BAUR}^{12}$ for some compounds. This method is limited by the restrictions of a point charge model.

12 W. H. BAur, Acta Cryst. 19, 909 [1965].
A direct determination of the hydrogen positions by neutron diffraction is not available. Further approximate informations are possible by low temperature deuteron magnetic resonance on $\mathrm{Na}_{2} \mathrm{~S}_{2} \mathrm{O}_{6}$. $2 \mathrm{D}_{2} \mathrm{O}$. By this method the principal axes of the field gradient tensor of the deuterons result. The main part of the electric field gradient in the heavy water molecules is given by electronic charge distribution of the OD bonds.

If we neglect the influence of the point charges surrounding the water molecules (which should be only a few percent of the total gradient) approximate values of the direction cosines of the OD bonds are available. We have undertaken such an experiment which is described and discussed in the following paper. Assuming that the structure of the deuterated compound is just the same as for the hydrated one a comparison of both compounds is possible. The direction cosines of the OD bonds were set to be equal to the main principal axis of the electric field gradient tensor. With a mean OD-bond length of $0.96 \AA$ the deuteron positions were calculated. A comparison of the deuteron positions with respect to the hydrogen coordinates, the $\mathrm{HH}$ distance and the bond angle $\mathrm{HOH}$ gained from the deuteron NQR experiments with the results of the method used in this paper is given in the following paper.

Acknowledgment: We are grateful to Dr. S. KETUdaT for interesting discussions and to the Deutsche Forschungsgemeinschaft for financial support. 